hep-th/0703031

SPhT-T07/029

NSF-KITP-07-29

\title{
Strong coupling limit of Bethe Ansatz equations
}

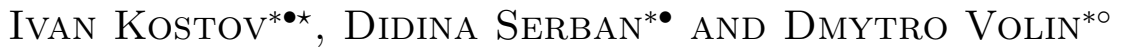 \\ * Service de Physique Théorique, CNRS-URA 2306 \\ C.E.A.-Saclay \\ F-91191 Gif-sur-Yvette, France \\ and \\ - Kavli Institute for Theoretical Physics, University of California \\ Santa Barbara, CA 93106 USA \\ and \\ - Bogolyubov Institute for Theoretical Physics \\ $14 b$ Metrolohichna Str. \\ Kyiv, 03143 Ukraine
}

\begin{abstract}
We develop a method to analyze the strong coupling limit of the Bethe ansatz equations supposed to give the spectrum of anomalous dimensions of the planar $\mathcal{N}=4$ gauge theory. This method is particularly adapted for the three rank-one sectors, su(2), su(1|1) and $s l(2)$. We use the elliptic parametrization of the Bethe ansatz variables, which degenerates to a hyperbolic one in the strong coupling limit. We analyze the equations for the highest excited states in the $s u(2)$ and $s u(1 \mid 1)$ sectors and for the state corresponding to the twist-two operator in the $\operatorname{sl}(2)$ sector, both without and with the dressing kernel. In some cases we were able to give analytic expressions for the leading order magnon densities. Our method reproduces all existing analytical and numerical results for these states at the leading order.
\end{abstract}

*Associate member of the Institute for Nuclear Research and Nuclear Energy, Bulgarian Academy of Sciences, 72 Tsarigradsko Chaussée, 1784 Sofia, Bulgaria 


\section{Introduction}

There is mounting evidence that the planar $\mathcal{N}=4$ SYM gauge theory is integrable [1, 2, 3] to higher orders in perturbation theory, nourishing the hope to prove by this mean its equivalence with the string theory on $A d S_{5} \times S^{5}$ [4, 5, 6]. Under the assumption of integrability, the spectrum of the anomalous dimensions of the planar $\mathcal{N}=4 \mathrm{SYM}$ gauge theory is encoded, at least for states with large number of operators, in the Bethe ansatz equations. The $S$-matrix behind these equations [7] is fixed by symmetry [8, 9], up to a scalar factor which is supposed to obey a crossing symmetry relation [10. At strong coupling, the scalar factor should allow to reproduce the string theory results. Its first two orders in the strong coupling expansion were fixed in this way [11, 12. More recently, based on previous work of Beisert, Hernández and López [13], Beisert, Eden and Staudacher [14] proposed an expression for the scalar factor which is crossing symmetric and reproduces both the strong coupling, string results and the perturbative results for the anomalous dimensions in the gauge theory. An efficient way of testing the predictions of the Bethe ansatz equations proved to be via the anomalous dimension of the twist-two operator. In the regime of large Lorentz spin $S$, this quantity scales logarithmically

$$
\Delta-S=f(g) \ln S+\ldots .
$$

The universal scaling function for $\mathcal{N}=4$ SYM up to third loop order was extracted [15] from a perturbative three-loop computation in QCD [16]. The same quantity appears in the iterative structure [17] of the multi-gluon amplitudes of the supersymmetric gauge theory. It was computed up to three loop order in [18, and, by an impressing effort, up to four loop order in [19, 20]. The four loop result agrees with the prediction based on the proposal [14]. On the other hand, at strong coupling the universal scaling function $f(g)$ is predicted to behave as $[21,22$.

$$
f(g)=4 g-\frac{3 \ln 2}{\pi}+\ldots .
$$

Here we adopt the recent convention for the coupling constant $g$

$$
g^{2}=\frac{\lambda}{16 \pi^{2}}
$$

After the proposal [14] was made, several groups [23, 24, 25] attempted to derive from it the strong coupling expression (1.2). While the numerical work [24] easily reproduced the behavior (1.2) and even predicted the next term in the expansion, the analytical treatment proved to be much more difficult. Up to now, only the first term was obtained analytically [23, 25].

One of the aspects which deserve further attention is to understand the origin of the dressing phase [14] and to test its validity. It is believed that the dressing phase comes from a non-trivial structure of the vacuum. Very recently, a structure similar to that of the dressing phase was obtained [26], via the nested Bethe ansatz, for one of the non-trivial "vacuum" states in one of the sectors which are not of rank one.

The aim of the present paper is to develop a systematic approach for solving the Bethe ansatz equations at strong coupling. Our analysis is based on the formulation of 
the Bethe equations as integral equations, and it works most simply on, although it is not restricted to, states as the antiferromagnetic state in the $s u(2)$ sector, on the most excited state in the $s u(1 \mid 1)$ sector, or for the finite-twist operators, in the $s l(2)$ sector. Some of this cases were already studied numerically or analytically; here we aim to a unitary treatment and hope that this method will be useful to obtain systematical $1 / \mathrm{g}$ expansion.

Our starting point is the elliptic parametrization of the variables $u$ and $x^{ \pm}$that appear in the Bethe equations. The elliptic parametrization we use here is related to the one proposed by Janik [10] by a Gauss-Landen transformation, and it appeared independently in [9] and [27]. The elliptic modulus is defined by

$$
\frac{k^{\prime}}{k}=\frac{1}{4 g} \equiv \epsilon
$$

In the hyperbolic limit $g \rightarrow \infty$, this parametrization supplies natural variables in the different regimes, recently discussed in [13, 28].

As it became clear from the numerical solutions of the integral equations [29], the points $u= \pm 2 g$ become singular for large $g$ and they split the real axis $u$ into two regions, with $|u| / 2 g$ less or larger than one, where two different hyperbolic parametrizations apply 1 The region $|u|<2 g$ is that of the "giant magnons", in the language of [28], and is characterized by finite values of the periodic momenta $p$. The region $|u|>2 g$ corresponds to the "plane-wave" limit [32], where the momenta are of the order $p \sim 1 / g$.

It is more difficult to see what happens exactly at the points $u= \pm 2 g$ and in their vicinity. These points separate the two regimes discussed above, and they are not properly described by either of the two parametrizations. In fact, they correspond to momenta of the order $p \sim g^{-1 / 2}$, or to the "near-flat space" region, again in the terminology of [28]. In some cases almost all the roots of the Bethe equations are concentrated in this region. It is possible to use the elliptic parametrization to obtain the strong coupling expansion in this regime 2 and it is clear that the expansion in this region involves powers of $g^{-1 / 2}$, or equivalently $1 / \lambda^{1 / 4}$.

In order to obtain the strong coupling limit of the dressing kernel [14] we have several options. One is to take the large coupling limit term by term in the series defining the dressing phase around $g=\infty$ [13, 14]. For the giant magnon and plane-wave limits this works, and this is probably the most straightforward way to obtained the leading orders. In the near-flat space limit, however, as it was noticed in [28], all the terms of the series contribute to the leading order in $1 / \mathrm{g}$. We would like to emphasize that, although the results for the leading order of the anomalous dimensions can be obtained without being particularly careful about the near-plane wave regime, the corrections will be crucially determined by it.

A second possibility is to use the representation of the dressing kernel given by the "magic formula" of [14], and this representation was already exploited in [25]. In this

\footnotetext{
${ }^{1}$ The two hyperbolic parametrizations are related by the transformation $s \rightarrow K-s$, where $K$ is the real period of the elliptic function.

${ }^{2}$ In this case, the parametrization is obtained by shifting $s \rightarrow \pm K / 2+s$.
} 
paper we give another related integral representation

$$
K_{d}\left(u, u^{\prime}\right)=4 \sum_{n \geq 1} \int_{-\infty}^{\infty} d v K_{-}^{1, n}(u, v)\left(K_{+}^{n,-1}\left(v, u^{\prime}\right)-K_{+}^{n, 1}\left(v, u^{\prime}\right)\right)
$$

where the kernels $K_{ \pm}\left(u, u^{\prime}\right)$ are the odd/even parts of the basic $s u(1 \mid 1)$ kernel $K\left(u, u^{\prime}\right)$, the inverse Fourier transform counterparts of the kernels $K_{0,1}\left(t, t^{\prime}\right)$ in the BES decomposition [14]. The upper indices $K_{ \pm}^{m, n}\left(u, u^{\prime}\right)$ mean essentially that the variables $u$ and $u^{\prime}$ are defined by elliptic functions of modulus

$$
\frac{k_{n}^{\prime}}{k_{n}}=\frac{n}{4 g}, \quad \frac{k_{m}^{\prime}}{k_{m}}=\frac{m}{4 g} .
$$

Similar variables were used in [13] and they are associated to bound states of magnons [35, 36]. The sum in (1.5) resembles to a sum over intermediate states; in particular, the energy associated to these "intermediate states" is of the type [35]

$$
E_{n}=\sqrt{n^{2}+16 g^{2} \sin ^{2} p / 2}
$$

In the large $g$ limit, the sum over $n$ can be taken with the help of the Euler-Maclaurin formula and the integrals over the variables $v$ and $z=n / 4 g$ are simple enough to be done. After taking the integrals, we reproduce the first term in the strong coupling expansion of the dressing kernel [11. Although technically this result is not very useful, it can be considered as another evidence that the weak coupling and strong coupling definitions of the dressing kernel [14] agree.

Turning to the explicit results, we obtain here the leading order result for anomalous dimensions of the highest excited state in the $s u(2)$ and $s u(1 \mid 1)$ sectors, both for the Bethe ansatz with the Beisert, Hernández, López/Beisert, Eden, Staudacher (BHL/BES) phase, and without it. Even if the kernel without the dressing phase is by now only of limited interest, we can still ask the question whether the $s u(1 \mid 1)$ sector arises from an effective model, in the same way the $s u(2)$ sector appears from the reduction of the Hubbard model at half-filling. The large $g$ limit of the $s u(1 \mid 1)$ sector should give a hint for the underlying degrees of freedom. In the $s u(2)$ sector, which was solved in [37, 38], the degrees of freedom are magnons, which become free as $g \rightarrow \infty$. Our result in the $s u(1 \mid 1)$ sector shows that these degrees of freedom do not correspond to a free system, and they are rather difficult to characterize. Two thirds of the excitations are of the type "giant magnon", with finite momenta, and one third are of the type "plane wave", with momenta of order $1 / \mathrm{g}$, see also the numerical results of [39]. We find for the energy without the dressing factor

$$
E_{s u(1 \mid 1)}=\frac{8 \ln 2}{\pi} g L, \quad E_{s u(2)}=\frac{4}{\pi} g L .
$$

The $s u(2)$ energy is the $g \rightarrow \infty$ limit of the exact result [37, 38]. When taking into account the BHL/BES phase, almost all roots of the Bethe equations, both for the $s u(2)$ and $s u(1 \mid 1)$ sector, fall into the region "near-flat space" regime, with momenta of order $1 / \sqrt{g}$. This phenomenon was first discussed in [11], where the $\lambda^{1 / 4}$ behavior was obtained 
from the BA equations. The integral equations are able to reproduce the leading result for the anomalous dimensions of the corresponding states, which were also obtained numerically and analytically in [39, 40]

$$
E_{s u(1 \mid 1)}^{d}=\sqrt{2 \pi g} L, \quad E_{s u(2)}^{d}=\sqrt{\pi g} L .
$$

We also analyzed the strong coupling limit of the equation derived by Eden and Staudacher (ES) [29] for the anomalous dimension of the twist-two operator without and with the dressing phase (BES equation). Similarly to the approaches [24, 23], we obtain that the strong coupling limit of the ES equation is pathological. The BES equation has a better behavior, and we are able to reproduce the leading term of the density obtained recently by Alday et al [25] using the Fourier space representation,

$$
\begin{gathered}
\sigma^{<}(u)=\frac{1}{4 \pi g^{2}} \quad \text { for } \quad|u|<2 g, \\
\sigma^{>}(u)=\frac{1}{4 \pi g^{2}}(1-\cosh s / 2) \quad \text { for } \quad u=2 g \operatorname{coth} s, \quad|u|>2 g .
\end{gathered}
$$

This solution reproduces the leading behavior of the universal scaling function (1.2).

For the next order in the density, an important fraction of the roots lie near the points $u \simeq \pm 2 g$. We show that without properly considering the scattering of the magnons in this region, the density is non-normalizable. We leave the fine analysis of the near-flat space region for future work.

The paper is organized as follows: in section 2 we write down the integral equations corresponding to the three sectors, in section 3 we present in detail the elliptic parametrization and we take the strong coupling limit of one of the the building blocks, the $s u(1 \mid 1)$ kernel. In section 4 we compute the energy of the highest states for the $s u(2)$ and $s u(1 \mid 1)$ without the dressing kernel and discuss the leading order solution of the Eden-Staudacher equation. In section 5 we give an integral representation of the dressing kernel and we check that it reproduces the already known results at strong coupling. We also give the strong coupling limit of the AFS phase [11]. The integral equations with the dressing phase equations are considered in section 6 .

\section{Bethe ansatz equations in integral form}

If we consider highly excited states, with a large number of magnons, the Bethe ansatz equations can be solved by transforming them into integral equations. These integral equations are particularly simple when the number of magnons is maximal, such that there are no holes in the magnon distribution 3 . Further simplification arise if we restrict ourselves to one of the three rank-one sectors, su(1|1), su(2) or $s l(2)$. The $s u(1 \mid 1)$ comprises one complex boson $Z$ and one fermion $U$, and the state with the maximum number of fermions

$$
\operatorname{Tr} U^{L}
$$

\footnotetext{
${ }^{3}$ The integral equation for the highest state in the $s u(1 \mid 1)$ sector was written down by one of us and M. Staudacher, 41] and in [4], the equation for the antiferromagnetic state in the $s u(2)$ sector was solved in [37], and the equation in $\operatorname{sl}(2)$ sector, or the Eden-Staudacher equation, was derived in [29].
} 
is, at least at weak coupling, the one with highest energy.

In the $s u(2)$ sector, corresponding to two complex bosons $Z$ and $X$, the state with highest energy is, again at small coupling $g$, the anti-ferromagnetic state. This is, in the case of even length, the singlet state

$$
\operatorname{Tr}(Z X)^{L / 2}+\ldots
$$

The $\operatorname{sl}(2)$ sector corresponds to combinations of one boson $Z$ and the covariant derivative $D$. The interesting operators are the so-called twist- $L$ operators with spin $S$,

$$
\operatorname{Tr} D^{S} Z^{L}
$$

The equations in the three rank-one sectors can be written in the compact form [8, 30]

$$
\left(\frac{x_{k}^{+}}{x_{k}^{-}}\right)^{L}=\prod_{l}^{M} \frac{1-g^{2} / x_{k}^{+} x_{l}^{-}}{1-g^{2} / x_{l}^{+} x_{k}^{-}}\left(\frac{x_{k}^{+}-x_{l}^{-}}{x_{k}^{-}-x_{l}^{+}}\right)^{\eta} e^{i \theta\left(u_{k}, u_{l}\right)},
$$

where $\eta=1,0,-1$ for $s u(2), s u(1 \mid 1)$ and $s l(2)$ respectively. The variables $x^{ \pm}$are defined by

$$
x(u)=\frac{1}{2} u\left(1+\sqrt{1-4 g^{2} / u^{2}}\right), \quad x^{ \pm}=x(u \pm i / 2) .
$$

It is often convenient to work with the momentum $p$, the physical variable of the magnon, defined by

$$
u(p)=\frac{1}{2} \cot \frac{p}{2} \sqrt{1+16 g^{2} \sin ^{2} \frac{p}{2}}, \quad \text { or } \quad x^{+} / x^{-}=e^{i p} .
$$

We are going to consider both the case where the phase $\theta\left(u_{k}, u_{l}\right)$ is zero and the case where it equals the BHL/BES dressing phase [13, 14].

Generically, the Bethe equations for the rank-one sectors can be written as

$$
e^{i p_{k} L}=\prod_{l=1}^{M} e^{i \varphi\left(u_{k}, u_{l}\right)}
$$

where $\varphi\left(u_{k}, u_{l}\right)$ is the scattering phase for two magnons. Let us exemplify the derivation of the integral equation on the $s u(1 \mid 1)$ case, which is the simplest one. We consider for convenience $L$ odd. The total number of magnons is $M=L$. When $g=0$, the equations (2.1) correspond to a free fermion system, with the occupation numbers

$$
p_{k}^{(0)}=2 \pi k / L, \quad k=-\frac{L-1}{2}, \ldots, \frac{L-1}{2} .
$$

When $g$ increases, the momenta start to evolve, according to the Bethe ansatz equations (2.1)

$$
\frac{p_{k}}{2 \pi}=\frac{k}{L}+\frac{1}{2 \pi L} \sum_{l=1}^{M} \varphi\left(u_{k}, u_{l}\right)
$$


When the length of the chain is very large, we can take the continuum limit. We introduce the variable $t=k / L$ and the density of rapidities $\rho(u)=-d t / d u$. The derivative with respect to $u$ of the equation (2.5) gives

$$
\rho(u)=-\frac{1}{2 \pi} \frac{d p}{d u}+\int_{-\infty}^{\infty} d u^{\prime} K\left(u, u^{\prime}\right) \rho\left(u^{\prime}\right),
$$

where the kernel is the derivative of the scattering phase

$$
K\left(u, u^{\prime}\right)=\frac{1}{2 \pi} \frac{d}{d u} \varphi\left(u, u^{\prime}\right)
$$

The derivation of the $s u(2)$ equation is similar, with the difference that the maximum number of magnons is $L / 2$ instead of $L$. This derivation follows closely the analysis of the antiferromagnetic state in the XXX model [42]. A more sophisticated treatment which is able to take into account the finite size corrections, was used in [43] to study the antiferromagnetic state in the BDS model [30] and in the Hubbard model.

The Bethe integral equations (2.1) can be formulated in terms of three basic kernels. The first one is the kernel $K\left(u, u^{\prime}\right)$, corresponding to the $s u(1 \mid 1)$ case without the dressing phase. The second one is the $s u(2)$ kernel $K_{s u(2)}\left(u, u^{\prime}\right)$, and the third one is the dressing kernel, corresponding to the dressing phase $\theta\left(u, u^{\prime}\right)$

$$
\begin{aligned}
K\left(u, u^{\prime}\right) & =\frac{1}{2 \pi i} \frac{d}{d u}\left(\ln \left(1-\frac{g^{2}}{x^{+}(u) x^{-}\left(u^{\prime}\right)}\right)-\ln \left(1-\frac{g^{2}}{x^{-}(u) x^{+}\left(u^{\prime}\right)}\right)\right) \\
K_{s u(2)}\left(u, u^{\prime}\right) & =\frac{1}{2 \pi i} \frac{d}{d u} \ln \left(\frac{u-u^{\prime}+i}{u-u^{\prime}-i}\right)=-\frac{1}{\pi} \frac{1}{\left(u-u^{\prime}\right)^{2}+1} \\
K_{d}\left(u, u^{\prime}\right) & =\frac{1}{2 \pi} \frac{d}{d u} \theta\left(u, u^{\prime}\right) .
\end{aligned}
$$

When the dressing phase is taken into account, the complete kernel is

$$
\begin{aligned}
\mathcal{K}_{\text {tot }}\left(u, u^{\prime}\right) & =(1-\eta) K\left(u, u^{\prime}\right)+\eta K_{s u(2)}\left(u, u^{\prime}\right)+K_{d}\left(u, u^{\prime}\right) \\
& \equiv \mathcal{K}\left(u, u^{\prime}\right)+\eta K_{s u(2)}\left(u, u^{\prime}\right)
\end{aligned}
$$

It will be sometimes convenient to work with the phase associated with the kernel $\mathcal{K}$

$$
\mathcal{K}\left(u, u^{\prime}\right)=\frac{1}{2 \pi} \partial_{u} \phi\left(u, u^{\prime}\right), \quad \phi=(1-\eta) \varphi+\theta .
$$

The $s l(2)$ sector is special in the sense that the number of magnons, also called spin, $M=S$, is not bounded, even if the length is finite ( $L=2$ for the twist-two operator). The lowest state with $S$ magnons can be characterized by the numbers

$$
n_{k}=k+\operatorname{sign}(k), \quad k=-(S-1) / 2, \ldots,(S-1) / 2 .
$$

The corresponding integral equation is given, again in the absence of the dressing phase, by

$$
\rho(u)=\frac{2}{s} \delta(u)+\frac{1}{\pi} \int_{-\infty}^{\infty} d u^{\prime} \frac{\rho\left(u^{\prime}\right)}{\left(u-u^{\prime}\right)^{2}+1}+2 \int_{-\infty}^{\infty} d u^{\prime} K\left(u, u^{\prime}\right) \rho\left(u^{\prime}\right),
$$


where the term proportional to $\delta(u)$ comes from the distribution of the mode numbers $n_{k}$. The limit $g=0$ of this equation is singular, and it was solved in [31]. The solution for large $S$ is

$$
\rho_{0}(u)=\frac{1}{\pi S} \ln \frac{1+\sqrt{1-4 u^{2} / S^{2}}}{1-\sqrt{1-4 u^{2} / S^{2}}}=\frac{2 \ln S}{\pi S}+\mathcal{O}(1 / S) .
$$

Eden and Staudacher [29] chose to separate the density $\rho(u)$ into the $g=0$ part, $\rho_{0}(u)$, and a fluctuation $\sigma(u)$

$$
\rho(u)=\rho_{0}(u)-2 g^{2} \frac{4 \ln S}{S} \sigma(u)+\mathcal{O}(1 / S)=-2 g^{2} \frac{4 \ln S}{S}\left(\sigma(u)-\frac{1}{4 \pi g^{2}}\right)+\mathcal{O}(1 / S) .
$$

From (2.14), the equation satisfied by the density fluctuation $\sigma(u)$ is

$$
\sigma(u)=\frac{1}{\pi} \int_{-\infty}^{\infty} d u^{\prime} \frac{\sigma\left(u^{\prime}\right)}{\left(u-u^{\prime}\right)^{2}+1}+2 \int_{-\infty}^{\infty} d u^{\prime} K\left(u, u^{\prime}\right)\left(\sigma\left(u^{\prime}\right)-\frac{1}{4 \pi g^{2}}\right) .
$$

This is essentially the equation derived by Eden and Staudacher [29], with the inhomogeneous term written differently. This way of writing has the advantage to show that the separation of $\rho(u)$ into $\rho_{0}(u)$ and $\sigma(u)$ makes sense, at large $g$, if $K\left(u, u^{\prime}\right)$ is sufficiently well behaved at large $u^{\prime}$. As we shall see, if $K\left(u, u^{\prime}\right)=\mathcal{O}(1)$ for large $g$ and $\left|u^{\prime}\right|>2 g$, the equation (2.17) will have only the trivial solution 4 , since at leading order in $1 / g$ one can write

$$
\int_{-\infty}^{\infty} d u^{\prime} K\left(u, u^{\prime}\right)\left(\sigma\left(u^{\prime}\right)-\frac{1}{4 \pi g^{2}}\right)=0 .
$$

In this case, the separation of the density into a free part and a perturbation is inconsistent. The Eden-Staudacher kernel is exactly of this type, and this might be the reason the strong coupling limit of the Eden-Staudacher equation is so pathological [23, 24]. The BES kernel, on the other hand, vanishes at the order $\mathcal{O}(1)$ for $\left|u^{\prime}\right|>2 g$, and the strong coupling limit of the solution of the BES equation is well behaved.

\section{$3 \quad$ Elliptic parametrization of the kernels}

\subsection{The elliptic map}

In the strong coupling regime, it is convenient to rescale the variables $u$ and $x^{ \pm}$in order to eliminate the overall factors of $g$. We will use the rescaled variables throughout the rest of the paper

$$
u=\frac{u_{\mathrm{old}}}{2 g}, \quad x^{ \pm}=\frac{x_{\mathrm{old}}^{ \pm}}{g}, \quad \epsilon=\frac{1}{4 g} .
$$

The function

$$
x(u)=u+\sqrt{u^{2}-1}
$$

has a branch cut along the interval $[-1,1]$. Since the Bethe equations involve the shifted variables $u \pm i \epsilon$, they contain two symmetric cuts, $[-1-i \epsilon, 1-i \epsilon]$ and $[-1+i \epsilon, 1+i \epsilon]$.

\footnotetext{
${ }^{4}$ At least if the kernel is non-degenerate.
} 
The cuts can be removed by introducing a global parametrization. We eliminate the parameter $u$ from

$$
u_{ \pm} \equiv u \pm i \epsilon=\frac{1}{2}\left(x^{ \pm}+1 / x^{ \pm}\right)
$$

to obtain the following relation between $x^{+}$and $x^{-}$

$$
\left(x^{+}-x^{-}\right)\left(1-\frac{1}{x^{+} x^{-}}\right)=4 i \epsilon .
$$

If we require that the momentum $p$ is real, then $x^{+}$and $x^{-}$are complex conjugate and $u$ real. Then the condition (3.4) for $x=x^{+}$and $\bar{x}=x^{-}$define a contour $\mathcal{C}$ in the complex $x$-plane. The contour consists, for $g$ real, of two connected components, $\mathcal{C}_{\infty}$ and $\mathcal{C}_{0}$, which are exchanged by the particle-antiparticle transformation $x \rightarrow 1 / x$. We denote by $\mathcal{C}_{\infty}$ the component that contains the point $x=\infty$; the other component contains the point $x=0$. In the limit $\epsilon \rightarrow 0$ the contours $\mathcal{C}_{\infty}$ and $\mathcal{C}_{0}$ develop cusps where they touch (Fig.1). At this point the contour $\mathcal{C}$ is the union of the real line and the unit circle. A second singular value is $\epsilon= \pm i$. Here the two connected component join into one (Fig.2).
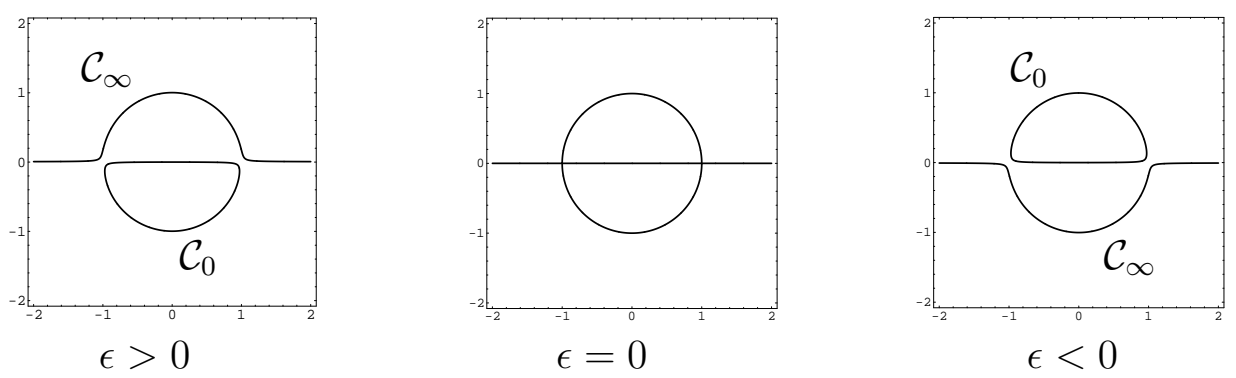

Fig.1 : The contour $\mathcal{C}$ in the $x$ plane for three real values of $\epsilon$ close to 0.
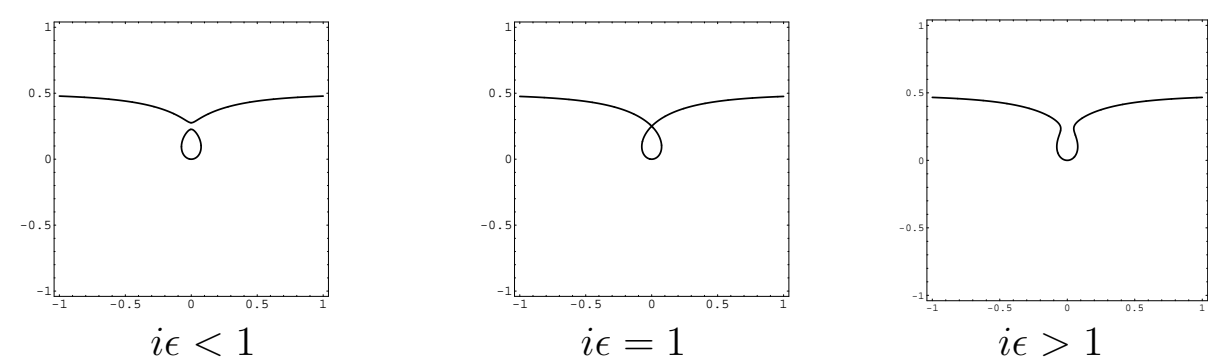

Fig. 2: The contour $\mathcal{C}$ for three imaginary values of $\epsilon$ close to $-i$.

The equation for the contour $\mathcal{C}$ can be written conveniently in terms of the momentum $p$ and a complementary parameter $\beta$

$$
x^{ \pm}=e^{(\beta \pm i p) / 2} .
$$


In these variables the relation (3.4) reads

$$
\sin \frac{p}{2} \sinh \frac{\beta}{2}=\epsilon
$$

and the parameter $u$ is expressed as

$$
u=\cos \frac{p}{2} \cosh \frac{\beta}{2} \text {. }
$$

In the following, we consider only positive values of $\epsilon$, and $p \in[0,2 \pi]$. This insures that $u$ in (3.7) takes all the real values once and only once.

The relation (3.6) is resolved by elliptic map5 with modulus $k$ defined as

$$
\epsilon=\frac{k^{\prime}}{k} \quad \text { or } \quad k=\frac{1}{\sqrt{1+\epsilon^{2}}} .
$$

Then $\beta$ and $p$ are parametrized by the Jacobi elliptic amplitude function

$$
p(s)=\pi-2 \operatorname{am}(\mathrm{K}-s, k), \quad \beta(s)=-i \pi-2 i \operatorname{am}\left(i \mathrm{~K}^{\prime}-s, k\right) .
$$

When the elliptic parameter $s$ sweeps the interval $[0,2 \mathrm{~K}]$, the momentum $p$ increases from 0 to $2 \pi$. The symmetries of $p$ and $\beta$ are

$$
\begin{gathered}
p(-s)=-p(s), \quad p(s+2 K)=p(s)+2 \pi \\
\beta(-s)=\beta(s)+2 \pi i, \quad \beta(2 K-s)=\beta(s) .
\end{gathered}
$$

The functions we will work with are actually expressed in terms of Jacobi elliptic functions

$$
\begin{array}{rlrl}
e^{ \pm \beta / 2} & =\frac{1 \pm \operatorname{dn} s}{k \operatorname{sn} s}, & e^{ \pm i p / 2}=\operatorname{cd} s \pm i k^{\prime} \operatorname{sd} s \\
\partial_{s} p & =2 k^{\prime} \operatorname{nd} s, & \partial_{s} \beta & =-2 \operatorname{cs} s .
\end{array}
$$

The contours $\mathcal{C}_{\infty}$ and $\mathcal{C}_{0}$ are parametrized respectively by $s \in I_{\infty}$ and $s \in I_{0}$, where

$$
I_{\infty}=[0,2 \mathrm{~K}], \quad I_{0}=\left[2 i \mathrm{~K}^{\prime}, 2 \mathrm{~K}+2 i \mathrm{~K}^{\prime}\right] .
$$

The original rapidity variable $u$ is parametrized as

$$
u(s)=\frac{1}{k} \frac{\operatorname{cn} s}{\operatorname{sn} s \operatorname{dn} s} .
$$

The map (3.14) obeys the symmetries $u(s)=-u(-s)=u(s+2 \mathrm{~K})=u\left(s+2 i \mathrm{~K}^{\prime}\right)$, as well as

$$
u(s) u(\mathrm{~K}-s)=1+\epsilon^{2} .
$$

\footnotetext{
${ }^{5}$ Our map is related to that of [10] by a Gauss-Landen transformation $k_{\text {Janik }}=2 \sqrt{k^{\prime}} /\left(1+k^{\prime}\right)$.
} 
The real axis in the $u$-plane is the image of the interval $I_{\infty}$. The functions $x^{ \pm}(s)$ are periodic in $s$ with periods $2 \mathrm{~K}$ and $4 i \mathrm{~K}^{\prime}$ and have the symmetries

$$
x^{+}(s)=x^{+}\left(\mathrm{K}-i \mathrm{~K}^{\prime}-s\right)=-x^{-}(-s), \quad x^{ \pm}\left(s+2 i \mathrm{~K}^{\prime}\right)=\frac{1}{x^{ \pm}(s)} .
$$

The first symmetry preserves the contours $\mathcal{C}_{\infty}$ and $\mathcal{C}_{0}$, while the second symmetry exchanges them. Note that the factors $e^{\beta / 2}$ and $e^{ \pm i p / 2}$ are themselves antiperiodic in $s \rightarrow s+2 \mathrm{~K}$. This is a little complication, due to the fact that we are working with trigonometric functions of $p / 2$, which are periodic with period $4 \pi$. However, in the physical variables, they always appear in combinations which are periodic with period $2 \pi$. As we already mentioned, we prefer to work with $p$ in the interval $(0,2 \pi)$.
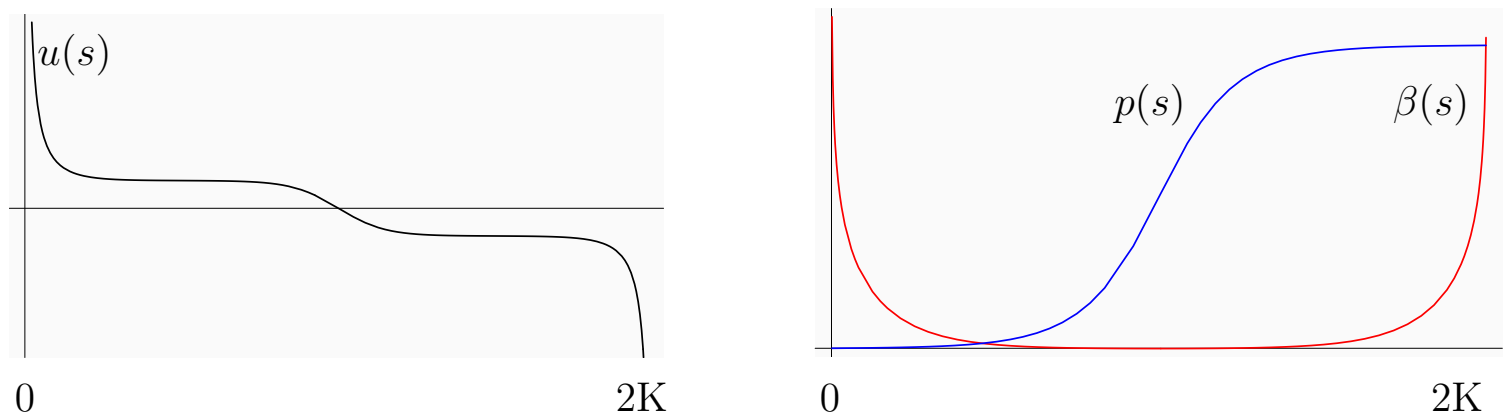

Fig. 3: Left: The function $u(s)$ for $\epsilon=10^{-3}$ on the interval $[0,2 \mathrm{~K}]$; Right: The functions $p(s)$ (in blue) and $\beta(s)$ (in red) for $\epsilon=10^{-3}$.

In the limit $\epsilon \rightarrow 0$, where $\mathrm{K} \rightarrow \infty$ and $\mathrm{K}^{\prime} \rightarrow \pi / 2$, the elliptic parametrization degenerates 6 and the variables $x$ and $u$ become hyperbolic functions of $s$. As the plot of $u(s)$ in Fig. 3 suggests, the parametrization interval splits into two different pieces, corresponding to two different regimes. By periodicity 7 , we can choose the parametrization interval as $\left[-\frac{1}{2} \mathrm{~K}, \frac{3}{2} \mathrm{~K}\right]$; then the first regime corresponds to the interval $\left[-\frac{1}{2} \mathrm{~K}, \frac{1}{2} \mathrm{~K}\right]$, which becomes the whole real axis when $\mathrm{K} \rightarrow \infty$. The second regime, corresponding to the interval $\left[\frac{1}{2} \mathrm{~K}, \frac{3}{2} \mathrm{~K}\right]$ can be mapped also to the interval $\left[-\frac{1}{2} \mathrm{~K}, \frac{1}{2} \mathrm{~K}\right]$ by the transformation

$$
s \rightarrow \mathrm{K}-s .
$$

The first interval corresponds to values $|u|>1$, while the second corresponds to $|u|<1$. We therefore introduce the suggestive notation

$$
I^{>}=\left[-\frac{1}{2} \mathrm{~K}, \frac{1}{2} \mathrm{~K}\right], \quad I^{<}=\left[\frac{1}{2} \mathrm{~K}, \frac{3}{2} \mathrm{~K}\right]
$$

\footnotetext{
${ }^{6}$ Another point where the torus degenerates into a cylinder is $\epsilon= \pm i$, or $g^{2}=-1 / 16$, cf. Fig.2.

${ }^{7}$ As already explained, the variables $u$ and $x$ are periodic with period $2 \mathrm{~K}$, and we can safely do the shift $s \rightarrow s-2 \mathrm{~K}$ for them, but for the variables $p, \beta$ we have to remember that the point $-\frac{1}{2} \mathrm{~K}$ was originally $\frac{3}{2} \mathrm{~K}$.
} 
and use the asymptotic formulas valid in the limit $\epsilon \rightarrow 0$ and for $s \in I^{>}$

$$
\begin{aligned}
\operatorname{sn} s & =\tanh s, & & \operatorname{sn}(\mathrm{K}-s)=1 \\
\operatorname{cn} s & =1 / \cosh s, & & \operatorname{cn}(\mathrm{K}-s)=k^{\prime} \sinh s, \\
\operatorname{dn} s & =1 / \cosh s, & & \operatorname{dn}(\mathrm{K}-s)=k^{\prime} \cosh s .
\end{aligned}
$$

The next terms in the expansion in $\epsilon$ are given in Appendix A.

In the hyperbolic limit, the expression (3.14) for $u$ becomes, in the two different regimes,

$$
u^{>}(s)=u(s) \simeq \operatorname{coth} s, \quad u^{<}(s)=u(\mathrm{~K}-s) \simeq \tanh s .
$$

The points $s= \pm \mathrm{K} / 2$, which are the fixed points of the transformation $s \rightarrow \mathrm{K}-s$, correspond to the points $u= \pm \sqrt{1+\epsilon^{2}}$. In the strong coupling limit, $\epsilon \rightarrow 0$, these are the points $u= \pm 1$, and they correspond to meeting points $s \rightarrow \pm \infty$ of the two parametrizations (3.20). These points will be very important, and we need a better parametrization for them. In the strong coupling limit the vicinity of these points is parametrized by (Appendix A)

$$
u\left( \pm \frac{1}{2} \mathrm{~K}+s\right)= \pm 1-\epsilon \sinh 2 s+\mathcal{O}\left(\epsilon^{2}\right) .
$$

The expressions for the momentum $p$ and its counterpart $\beta$ in these regimes are

$$
\sinh \beta / 2=\sqrt{\epsilon} e^{\mp s} \quad \sin p / 2=\sqrt{\epsilon} e^{ \pm s},
$$

where the upper sign correspond to momenta close to 0 and the lower sign to momenta close to $2 \pi$. A similar parametrization was used in [28].

\subsection{Strong coupling limit of the $s u(1 \mid 1)$ kernel}

We saw that in the strong coupling limit the integration interval splits naturally into two domains, $|u|<1$ and $|u|>1$, which are the images of the intervals $I^{<}$and $I^{>}$in the $s$ parametrization. As it is clear from Fig. 1, in the first domain the complex variable $x=x^{+}$becomes asymptotically unimodular, while in the second domain it becomes asymptotically real. For $\epsilon \rightarrow 0$ the relations (3.6) and (3.7) give

$$
\begin{aligned}
& \beta \rightarrow 0, \quad u=\cos p / 2 \quad \text { if }|u|<1, \\
& p \rightarrow 0, \quad u=\cosh \beta / 2 \text { if }|u|>1 \text {. }
\end{aligned}
$$

Passing to the elliptic parameter $s$ we write the integral equation (2.6) as

$$
\rho(s)=\frac{1}{2 \pi} p^{\prime}(s)+\int_{I_{\infty}} d s_{1} K\left(s, s_{1}\right) \rho\left(s_{1}\right)
$$

where

$$
\rho(s)=\left|u^{\prime}(s)\right| \rho(u), \quad K\left(s, s_{1}\right)=\left|u^{\prime}(s)\right| K\left(u, u_{1}\right) .
$$

The first term in the r.h.s. of (3.25) changes sign because the derivative $u^{\prime}(s)$ is negative.

The kernel is equal to the derivative

$$
K\left(s, s_{1}\right)=-\frac{1}{2 \pi i} \frac{d}{d s}\left[\ln \left(1-e^{-\frac{1}{2}\left(\beta+\beta_{1}\right)} e^{-i \frac{1}{2}\left(p-p_{1}\right)}\right)-\ln \left(1-e^{-\frac{1}{2}\left(\beta+\beta_{1}\right)} e^{i \frac{1}{2}\left(p-p_{1}\right)}\right)\right]
$$




$$
=\frac{1}{4 \pi} p^{\prime}(s)-\frac{1}{4 \pi} \frac{p^{\prime}(s) \sinh \frac{1}{2}\left(\beta+\beta_{1}\right)-\beta^{\prime}(s) \sin \frac{1}{2}\left(p-p_{1}\right)}{\cosh \frac{1}{2}\left(\beta+\beta_{1}\right)-\cos \frac{1}{2}\left(p-p_{1}\right)} .
$$

Let us mention here that the piece $p^{\prime}(s) / 4 \pi$ has a physical meaning: it corresponds to a change of the effective length of the chain. Every magnon increases the effective length of the chain by $1 / 2$. This is particularly clear on the discrete equations, where, at strong coupling, the Bethe equations become, as it was noticed in [44]

$$
e^{i p_{k}(L+M / 2)}=1
$$

under the condition that all $p_{k}$ are finite.

It is now easy to take the strong coupling limit using the asymptotic expressions for $p(s)$ and $\beta(s)$, which can be found in Appendix A. The asymptotic form of the kernel is given by four different analytic expressions depending on whether its arguments are in the interval $I^{>}$or $I<$. After applying the redefinition (3.17) to the arguments that belong to the second interval, we can write the result as a $2 \times 2$ matrix kernel

$$
\left(\begin{array}{ll}
K^{>>}\left(s, s_{1}\right) & K^{><}\left(s, s_{1}\right) \\
K^{<>}\left(s, s_{1}\right) & K^{<<}\left(s, s_{1}\right)
\end{array}\right):=\left(\begin{array}{ll}
K\left(s, s_{1}\right) & K\left(s, \mathrm{~K}-s_{1}\right) \\
K\left(\mathrm{~K}-s, s_{1}\right) & K\left(\mathrm{~K}-s, \mathrm{~K}-s_{1}\right)
\end{array}\right) .
$$

The arguments $s, s_{1}$ are defined in the interval $I^{>}$, which extends to $[-\infty, \infty]$ when $\epsilon \rightarrow 0$. Similarly the density splits into two components,

$$
\left(\begin{array}{l}
\rho^{>}(s) \\
\rho^{<}(s)
\end{array}\right)=\left(\begin{array}{l}
\rho(s) \\
\rho(\mathrm{K}-s)
\end{array}\right)
$$

satisfying, in the limit $\epsilon \rightarrow 0$, the normalization condition 8

$$
\int_{-\infty}^{\infty} d s \rho^{<}+\int_{-\infty}^{\infty} d s \rho^{>}=1
$$

Eq. (3.25) now takes the form

$$
\begin{aligned}
& \rho^{>}(s)=\int_{-\infty}^{\infty} d s_{1} K^{>>}\left(s, s_{1}\right) \rho^{>}\left(s_{1}\right)+\int_{-\infty}^{\infty} d s_{1} K^{><}\left(s, s_{1}\right) \rho^{<}\left(s_{1}\right) \\
& \rho^{<}(s)=\frac{p^{\prime}(s)}{2 \pi}+\int_{-\infty}^{\infty} d s_{1} K^{<>}\left(s, s_{1}\right) \rho^{>}\left(s_{1}\right)+\int_{-\infty}^{\infty} d s_{1} K^{<<}\left(s, s_{1}\right) \rho^{<}\left(s_{1}\right) .
\end{aligned}
$$

The matrix elements of the kernel (3.29), evaluated in Appendix A, are

$$
\begin{aligned}
K^{<<}\left(s, s^{\prime}\right) & =\frac{1}{2 \pi} \frac{1}{\cosh s}-\delta\left(s-s^{\prime}\right), \\
K^{<>}\left(s, s^{\prime}\right) & =\frac{1}{2 \pi} \frac{1}{\cosh s}-\frac{1}{2 \pi} \frac{1}{\cosh \left(s-s^{\prime}\right)}, \\
K^{><}\left(s, s^{\prime}\right) & =\frac{1}{2 \pi} \frac{1}{\cosh \left(s-s^{\prime}\right)}, \\
K^{>>}\left(s, s^{\prime \prime}\right) & =0 .
\end{aligned}
$$

\footnotetext{
${ }^{8}$ This is true only for the kernel without the dressing phase, at the leading order.
} 
Note that in the hyperbolic limit all four integration kernels depend on the difference of the arguments. This is a considerable simplification, since the equation can be now solved using Fourier transform.

In the third regime, which corresponds to the points around $u= \pm 1$ the momenta are parametrized by (3.22). This regime will be very important for evaluation of the density with the BHL/BES kernel. The appropriate parametrization is given by (3.21) and (3.22). The only component of the undressed kernel that survives at the leading order is when either $u, u^{\prime} \simeq 1$ or $u, u^{\prime} \simeq-1$

$$
\begin{aligned}
& K^{++}\left(s, s^{\prime}\right)=K^{--}\left(-s,-s^{\prime}\right) \\
& =-\frac{1}{4 \pi} \frac{\sinh \left(s-s^{\prime}\right)+1}{\cosh \left(s-s^{\prime}\right) \cosh \left(s+s^{\prime}\right)-\sinh \left(s+s^{\prime}\right)}+\mathcal{O}(\sqrt{\epsilon}) .
\end{aligned}
$$

If only one of the arguments is in the intermediate region, the kernel is of order $\sqrt{\epsilon}$.

One might argue that there are many intermediate regimes, where $p$ scales like an arbitrary power of $\epsilon$,

$$
p \sim \epsilon^{1-\gamma}, \quad \beta \sim \epsilon^{\gamma} \quad \gamma \in(0,1) .
$$

However, if $\gamma \neq 1 / 2$, one of the variables $p$ and $\beta$ dominates the other, and these regimes are properly taken into account in the regions $\underset{<}{>}$. Only for $\gamma=1 / 2$ the quantities $p$ and $\beta$ are of the same order of magnitude and this is why we have to consider this case separately.

\section{Solving the integral equations in the strong cou- pling limit without the dressing kernel}

\subsection{The $s u(2)$ case}

The $s u(2)$ kernel is the only one which is of difference form, therefore it can be exactly solved for any value of $g$ [37, 38]. The energy of the antiferromagnetic state is identical to the energy of the ground state for the Hubbard model at half filling [46]. Here, we would like to comment on the strong coupling limit of this solution. The integral equation is particularly simple, since the kernel becomes simply the delta function

$$
K_{s u(2)}\left(u, u^{\prime}\right)=-\lim _{\epsilon \rightarrow 0} \frac{1}{\pi} \frac{2 \epsilon}{\left(u-u^{\prime}\right)^{2}+4 \epsilon^{2}}=-\delta\left(u-u^{\prime}\right) .
$$

The integral equation reduces then to

$$
2 \rho(u)=-\frac{1}{2 \pi} \frac{d p}{d u}, \quad \text { or } \quad \rho(p)=\frac{1}{4 \pi} .
$$

The solution corresponds to $L / 2$ roots distributed uniformly between $p=0$ and $2 \pi$. In this case, the density is normalized to $1 / 2$, because the total number of magnons is $L / 2$. All roots of the Bethe equations are inside the interval $|u|<1$ and the energy is

$$
E_{s u(2)}=4 g L \int_{0}^{2 \pi} d p|\sin p / 2| \rho(p)=\frac{4 g L}{\pi} .
$$


Physically, the excitations are the (usual) magnons. The solution obtained above shows that, in the strong coupling limit, the magnons are free, except for the statistical repulsion (4.1). The situation is very much similar to that of the Haldane-Shastry model [47, 48], where the magnons are also free up to the statistical repulsion, translated into the rule that two magnons cannot occupy successive momenta. Let us remind that the scattering phase for particles with purely statistical interaction is [49]

$$
\varphi\left(p, p^{\prime}\right)=(\lambda-1) \pi \operatorname{sign}\left(p-p^{\prime}\right),
$$

which is consistent with (4.1), the statistical parameter of the magnons being $\lambda=2$. The only difference with the Haldane-Shastry spin chain is that here the magnons have the dispersion relation

$$
E(p)=4 g|\sin p / 2|,
$$

which is typical for a finite-difference hamiltonian, while the Haldane-Shastry magnons have the dispersion relation

$$
E_{H S}(p)=p(2 \pi-p) .
$$

A natural candidate for a model with purely statistical interaction and with trigonometric dispersion relation is the Ruijenaars-Schneider model [50]. This suggests the existence of a spin model which would describe the spin sector of the half-filled Hubbard model for any value of $g$ and which might be a multi-spin deformation of the Inozemtsev model [51, 52].

\subsection{The $s u(1 \mid 1)$ case}

The solution of the $s u(1 \mid 1)$ sector is considerably more involved, but its leading order still can be obtained in closed form. Since now the roots of the Bethe equations occur both in the regions $|u|<1$ and $|u|>1$, the integral equation splits up into two coupled equations for the densities in the two regions

$$
\begin{aligned}
2 \rho^{<}(s) & =\frac{3}{2 \pi} \frac{1}{\cosh s}-\frac{1}{2 \pi} \int_{-\infty}^{\infty} d s^{\prime} \frac{\rho^{>}\left(s^{\prime}\right)}{\cosh \left(s-s^{\prime}\right)}, \\
\rho^{>}(s) & =\frac{1}{2 \pi} \int_{-\infty}^{\infty} d s^{\prime} \frac{\rho^{<}\left(s^{\prime}\right)}{\cosh \left(s-s^{\prime}\right)} .
\end{aligned}
$$

Substituting the second equation into the first we obtain a new equation where the kernel is of difference form

$$
\rho^{<}(s)=\frac{3}{4 \pi} \frac{1}{\cosh s}-\frac{1}{4 \pi^{2}} \int_{-\infty}^{\infty} d s^{\prime} \frac{s-s^{\prime}}{\sinh \left(s-s^{\prime}\right)} \rho^{<}\left(s^{\prime}\right),
$$

and therefore is solved by Fourier transformation. In Fourier space the equation becomes algebraic 9

$$
\rho^{<}(t)=\frac{3}{4 \pi} \frac{\pi}{\cosh (\pi t / 2)}-\frac{1}{4 \pi^{2}} \frac{\pi^{2}}{2 \cosh ^{2}(\pi t / 2)} \rho^{<}(t),
$$

\footnotetext{
${ }^{9}$ In order to keep the notation simple, we are going to use the same symbol for the Fourier transforms $\rho^{<}(t), \rho^{>}(t)$ of the densities.
} 
with the solution

$$
\rho^{<}(t)=\frac{6 \cosh (\pi t / 2)}{1+8 \cosh ^{2}(\pi t / 2)}
$$

Transforming back to the $s$-variable, we get

$$
\begin{aligned}
\rho^{<}(s) & =\frac{1}{\sqrt{2} \pi} \frac{\cos 2 a s / \pi}{\cosh s}, \\
a & =\ln \sqrt{2} \simeq .34657 .
\end{aligned}
$$

For the density of roots $\rho(u)$ we obtain in the interval $|u|<1$

$$
\rho^{<}(u)=\frac{\rho^{<}(s)}{\left|u^{\prime}(s)\right|}=\frac{1}{\sqrt{2} \pi} \frac{1}{\sqrt{1-u^{2}}} \cos \left(\frac{a}{\pi} \ln \frac{1+u}{1-u}\right) .
$$

To evaluate the function $\rho^{>}(u)$, we make the change of variable $u=\operatorname{coth} s$, which is appropriate for $|u|>1$, so that we get

$$
\rho^{>}(s)=\frac{1}{2 \sqrt{2} \pi^{2}} \int_{-\infty}^{\infty} \frac{d s^{\prime}}{\cosh s^{\prime}} \frac{\cos \left(2 a s^{\prime} / \pi\right)}{\cosh \left(s-s^{\prime}\right)}=\frac{1}{\pi} \frac{\sin (2 a s / \pi)}{\sinh s},
$$

or, in the initial variables,

$$
\rho^{>}(u)=\frac{1}{\pi} \frac{1}{\sqrt{u^{2}-1}} \sin \left(\frac{a}{\pi} \ln \frac{u+1}{u-1}\right) .
$$

The result shows a singularity at the points $u= \pm 1$; moreover, it can take negative values. This happens at values of $1-u \simeq 10^{-5}$ and is presumably due to the roots around $u= \pm 1$ which were not taken properly into account. Except for these oscillations, the result reproduces nicely the solution obtained by numerical integration of the Fredholm equation 10 .

The total normalization of the density is insured, since $\int_{<} d u \rho_{<}(u)=2 / 3$ and $\int_{>} d u \rho_{>}(u)=1 / 3$. So two third of the roots of the Bethe equations fall in the region $|u|<1$ and one third in the intervals $|u|>1$. This phenomenon is also present in the numerical solution of [39]. Finally, the energy of the state is given by

$$
\begin{aligned}
E_{s u(1 \mid 1)} & =4 g L \int_{-1}^{1} d u \rho_{<}(u) \sqrt{1-u^{2}} \\
& =\frac{2 \sqrt{2} g L}{\pi} \int_{-\infty}^{\infty} d s \frac{\cos 2 a s / \pi}{\cosh ^{2} s}=\frac{16 a}{\pi} g L
\end{aligned}
$$

This result is consistent with that of Beccaria and Del Debbio 39]. We obtain for their constant $c_{L}$ the value $c_{L}=2 \ln (2) / \pi^{2} \simeq .14046$.

\footnotetext{
${ }^{10}$ We thank M. Staudacher for supplying us the solution obtained by numerical integration.
} 


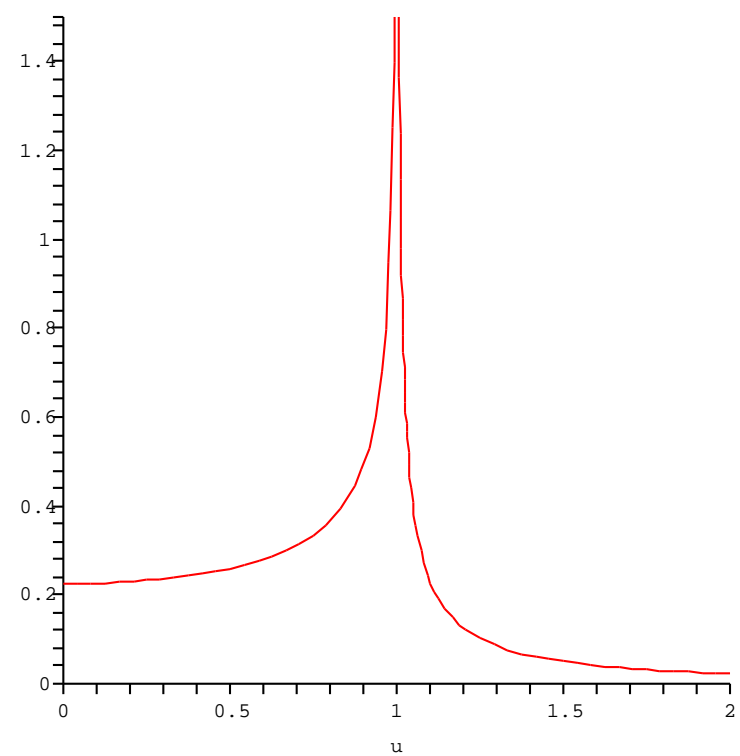

Fig. 4: Density profile predicted by the analytic solution (4.14), (4.16). Only the $u>0$ part is shown. The oscillations do not show yet at the chosen scale.

\subsection{The $\operatorname{sl}(2)$ case}

The dimension of the twist two operator was originally supposed to be determined by the solution of Eden-Staudacher equation [29]

$$
\sigma(u)=\frac{1}{\pi} \int_{-\infty}^{\infty} d u^{\prime} \frac{2 \epsilon}{\left(u-u^{\prime}\right)^{2}+4 \epsilon^{2}} \sigma\left(u^{\prime}\right)+2 \int d u^{\prime} K\left(u, u^{\prime}\right)\left(\sigma\left(u^{\prime}\right)-\frac{1}{2 \pi g}\right) .
$$

The anomalous dimension is simply given by the normalization of the density fluctuation $\sigma(u) 53$

$$
f(g)=16 g^{2} \int_{-\infty}^{\infty} d u \sigma(u),
$$

or alternatively by the formula 29

$$
f(g)=8 g^{2}\left(1-2 g \int_{-\infty}^{\infty} d u \sigma(u)\left(\frac{i}{x^{+}(u)}-\frac{i}{x^{-}(u)}\right)\right) .
$$

As explained in section 2, in the limit $\epsilon=1 / 4 g \rightarrow 0$ the equation (4.19) becomes

$$
\int d u^{\prime} K\left(u, u^{\prime}\right)\left(\sigma\left(u^{\prime}\right)-\frac{1}{2 \pi g}\right)=0 .
$$

Obviously, this equation has as solution 11

$$
\sigma(u)=\frac{1}{2 \pi g}
$$

\footnotetext{
${ }^{11}$ Here we use the variable $u$ rescaled by $2 g$, whence the extra factors of $2 g$ in the density.
} 
on the whole real axis. This solution, first obtained in [29], seems to be correct in the interval $|u|<1$, since it insures the vanishing of the $\mathcal{O}\left(g^{2}\right)$ term in $f(g)$

$$
f(g)=8 g^{2}\left(1-4 g \int_{-1}^{1} d u \sigma^{<}(u) \sqrt{1-u^{2}}+\mathcal{O}(1 / g)\right)=\mathcal{O}(g) .
$$

In the interval $|u|>1$, although it has the right scaling in $g$, the solution (4.23) is not integrable and it gives formally an infinite value for the anomalous dimension of the twist-two operator. As mentioned in the section 2, the density (4.23) exactly compensate the leading order in $S$ in $\rho_{0}(u)$ everywhere in $u$

$$
\rho_{0}(u)=\frac{4 g}{\pi} \frac{\ln S}{S}+\ldots .
$$

Unfortunately, as we show below, (4.23) is the unique solution to the ES equation at strong coupling. We can write the equations as

$$
\begin{aligned}
\frac{1}{2 \pi} \int d s^{\prime} \frac{\sigma^{<}\left(s^{\prime}\right)}{\cosh \left(s-s^{\prime}\right)} & =\frac{1}{8 \pi g} \frac{1}{\cosh ^{2}(s / 2)}, \\
\frac{1}{2 \pi} \int d s^{\prime} \frac{\sigma^{>}\left(s^{\prime}\right)}{\cosh \left(s-s^{\prime}\right)}+\sigma^{<}(s) & =\frac{1}{4 \pi g} \frac{1}{\cosh ^{2}(s)}+\frac{1}{2 \pi} \frac{A}{\cosh (s)},
\end{aligned}
$$

where $A=\int d s\left(\sigma^{<}(s)+\sigma^{>}(s)\right)$ is the normalization of the density. In Fourier transformed form, the equations become

$$
\begin{aligned}
\frac{\sigma^{<}(t)}{2 \cosh \pi t / 2} & =\frac{t}{2 g \sinh \pi t}, \\
\frac{\sigma^{>}(t)}{2 \cosh \pi t / 2}+\sigma^{<}(t) & =\frac{t}{4 g \sinh \pi t / 2}+\frac{A}{2 \cosh \pi t / 2} .
\end{aligned}
$$

The first line is readily solved as

$$
\sigma^{<}(t)=\frac{1}{2 g \sinh \pi t / 2}, \quad \text { or } \quad \sigma^{<}(s)=\frac{1}{2 \pi g} \frac{1}{\cosh ^{2} s}, \quad \text { or } \quad \sigma^{<}(u)=\frac{1}{2 \pi g},
$$

while the solution for the second is

$$
\begin{gathered}
\sigma^{>}(t)=A-\frac{t}{2 g \pi \tanh \pi t / 2}, \quad \text { or } \quad \sigma^{>}(s)=A \delta(s)+\frac{1}{2 \pi g} \frac{1}{\sinh ^{2} s}, \\
\text { or } \quad \sigma^{>}(u)=-A \delta(u-\infty)+\frac{1}{2 \pi g} .
\end{gathered}
$$

The normalization $A$ is not fixed by the equation.

All other approaches tried to compute the strong coupling limit from the EdenStaudacher ran into some pathology as well: the result obtained by Kotikov and Lipatov [23] oscillates strongly, the result obtained numerically in [24] may not converge to a straight line in $g$ as expected 12 and the numerical solution for $\sigma(u)$ in 29 does not seem to converge to a value with definite normalization when $g \rightarrow \infty$. We believe that all these pathologies are related to the separation of the density $\rho(u)$ into $\rho_{0}(u)$ and $\sigma(u)$. Rather surprisingly, this pathology disappear when the dressing kernel is taken into account, as we will show in the section 6 .

\footnotetext{
${ }^{12}$ We thank the authors of [24] for checking the behavior of their ES solution.
} 


\section{$5 \quad$ The dressing kernel}

\subsection{Integral representation}

Beisert, Eden and Staudacher proposed an expression for the dressing factor in the Bethe ansatz equations. This dressing factor translates into a correction to the kernel in the integral equation. Their formula is given in Fourier transformed form; for our purposes an expression in terms of variables $u$ is more suited. Let us start with the Fourier transform of the $s u(1 \mid 1)$ kerne 13

$$
\hat{K}\left(t, t^{\prime}\right)=-\pi\left(1-\operatorname{sign} t t^{\prime}\right)|t| e^{-\left(|t|+\left|t^{\prime}\right|\right) \epsilon} \sum_{n>0} n \frac{J_{n}(|t|) J_{n}\left(\left|t^{\prime}\right|\right)}{\left|t t^{\prime}\right|} .
$$

which is related to the kernel of [14] by

$$
\hat{K}\left(t, t^{\prime}\right)=-\frac{\pi}{2}\left(1-\operatorname{sign} t t^{\prime}\right)|t| e^{-\left(|t|+\left|t^{\prime}\right|\right) \epsilon} \hat{K}_{m}\left(|t|,\left|t^{\prime}\right|\right)
$$

and can be broken into a symmetric and antisymmetric part

$$
\begin{aligned}
\hat{K}\left(t, t^{\prime}\right) & =\hat{K}_{+}\left(t, t^{\prime}\right)+\hat{K}_{-}\left(t, t^{\prime}\right) \\
& =-\frac{\pi}{2}\left(1-\operatorname{sign} t t^{\prime}\right)|t| e^{-\left(|t|+\left|t^{\prime}\right|\right) \epsilon}\left(K_{0}\left(|t|,\left|t^{\prime}\right|\right)+K_{1}\left(|t|,\left|t^{\prime}\right|\right)\right) .
\end{aligned}
$$

In the ES and BES equations, the kernel is written in the Fourier variables defined only for positive values of $t$ and $t^{\prime}$. This is made possible since the densities $\rho(u)$ and $\sigma(u)$ are symmetric under $u \rightarrow-u$, and therefore under $t \rightarrow-t$, for the states under consideration. For generic states, this symmetry is not present, and this is why it is desirable to have a non symmetric kernel at hand. The extension of $K_{d}\left(t, t^{\prime}\right)$ to negative values of $t$ and $t^{\prime}$ is ambiguous, and undoing the Fourier transform in the "magic" formula of [14] will be ambiguous as well.

The authors of [25] gave a prescription to to define $K_{d}\left(u, u^{\prime}\right)$ by symmetrizing all the kernels on the second variable $K\left(u, u^{\prime}\right) \rightarrow \frac{1}{2}\left(K\left(u, u^{\prime}\right)+K\left(u,-u^{\prime}\right)\right)$. We try to avoid symmetrizing the kernels from the beginning, and give here a different prescription, using instead that the full BES kernel is antisymmetric with respect to $u_{ \pm} \rightarrow u_{\mp}$ on each variable separately. We remind that the transformations $u \rightarrow-u$ and $u_{ \pm} \rightarrow u_{\mp}$ translate in the following way on the variable $p$

$$
\begin{array}{rll}
u \rightarrow-u & \Leftrightarrow & p \rightarrow 2 \pi-p \\
u_{ \pm} \rightarrow u_{\mp} & \Leftrightarrow & p \rightarrow-p
\end{array}
$$

while the variable $\beta$ stays unchanged. Of course, these two transformations act differently on the Fourier transform.

It will be simpler to work with the non-symmetrized kernels and impose the antisymmetry under (5.3) at the end of the computation. We define, for $t, t^{\prime}>0$,

$$
\hat{K}_{d}\left(t,-t^{\prime}\right) \equiv-4 \pi t e^{-\left(t+t^{\prime}\right) \epsilon} \hat{K}_{c}\left(t, t^{\prime}\right)
$$

\footnotetext{
${ }^{13}$ Our definition of the kernel differs by a minus sign from the one of [29].
} 


$$
\begin{aligned}
& =-4 \pi t e^{-\left(t+t^{\prime}\right) \epsilon} \int_{0}^{\infty} d t^{\prime \prime} \hat{K}_{1}\left(t, t^{\prime \prime}\right) \frac{t^{\prime \prime}}{e^{2 \epsilon t^{\prime \prime}}-1} \hat{K}_{0}\left(t^{\prime \prime}, t^{\prime}\right) \\
& =-\frac{4}{\pi} \int_{-\infty}^{\infty} d t^{\prime \prime} \hat{K}_{-}\left(t,-t^{\prime \prime}\right) \frac{e^{2 \epsilon\left|t^{\prime \prime}\right|}}{e^{2 \epsilon\left|t^{\prime \prime}\right|}-1} \hat{K}_{+}\left(t^{\prime \prime},-t^{\prime}\right) .
\end{aligned}
$$

For any values of $t, t^{\prime}$, we can define the dressing kernel as a convolution

$$
\hat{K}_{d}\left(t, t^{\prime}\right)=-\frac{4}{\pi} \int_{-\infty}^{\infty} d t^{\prime \prime} \hat{K}_{-}\left(t, t^{\prime \prime}\right) \frac{e^{2 \epsilon\left|t^{\prime \prime}\right|}}{e^{2 \epsilon\left|t^{\prime \prime}\right|}-1} \hat{K}_{+}\left(-t^{\prime \prime}, t^{\prime}\right)
$$

The dressing kernel $\hat{K}_{d}\left(t, t^{\prime}\right)$, defined in this way, is proportional to $1-\operatorname{sign} t t^{\prime}$. Note, however, that after anti-symmetrization under (5.3) this property will not hold anymore. Going back to the $u$ variables we obtain

$$
K_{d}\left(u, u^{\prime}\right)=-8 \int_{-\infty}^{\infty} d v K_{-}(u, v) K_{+}\left(v, u^{\prime}\right)-\frac{4}{\epsilon} \int_{-\infty}^{\infty} d v d v^{\prime} K_{-}(u, v) h\left(v-v^{\prime}\right) \Theta_{+}\left(v^{\prime}, u^{\prime}\right)
$$

where

$$
h(u)=\frac{2 \epsilon}{2 \pi} \int_{-\infty}^{\infty} d t \frac{|t| e^{i t u}}{e^{2 \epsilon|t|}-1}=\frac{2 \epsilon}{2 \pi}\left(\frac{1}{u^{2}}-\frac{(\pi / 2 \epsilon)^{2}}{\sinh ^{2}(\pi u / 2 \epsilon)}\right)
$$

and $\Theta_{+}\left(u, u^{\prime}\right)$ is the Fourier transform of $\hat{K}_{+}\left(t, t^{\prime}\right) /|t|$. The function $h(u)$ becomes $\delta(u)$ as $\epsilon$ approaches 0. Since the kernels involved in the convolution in (5.5) are of order $\epsilon^{0}$, the dressing kernel will be of order $\epsilon^{-1}$. This formula is very close to the one in [25], except that we traded the difficulty in evaluating the function $h(u)$ for the difficulty in evaluating the phase $\Theta_{+}(u, v)$.

Let us now write down the expressions of $K_{+}\left(u, u^{\prime}\right)$ and $K_{-}\left(u, u^{\prime}\right)$, which contain the odd, respectively even powers in the expansion of the logarithm

$$
\begin{aligned}
& K_{-}(u, v)=\frac{1}{4 \pi i} \partial_{u} \ln \frac{\left(1-X_{+-}\right)\left(1+X_{+-}\right)}{\left(1-X_{-+}\right)\left(1+X_{-+}\right)}, \\
& K_{+}(u, v)=\frac{1}{4 \pi i} \partial_{u} \ln \frac{\left(1-X_{+-}\right)\left(1+X_{-+}\right)}{\left(1+X_{+-}\right)\left(1-X_{-+}\right)}, \\
& \Theta_{+}(u, v)=-\frac{1}{4 \pi} \ln \frac{\left(1-X_{+-}\right)\left(1-X_{-+}\right)}{\left(1+X_{+-}\right)\left(1+X_{-+}\right)},
\end{aligned}
$$

with

$$
X_{+-}=\frac{1}{x^{+}(u) x^{-}(v)}, \quad X_{-+}=\frac{1}{x^{-}(u) x^{+}(v)} .
$$

A strategy to compute the expansion of $K_{d}$ in the strong coupling limit is to compute the limit of the auxiliary kernels (5.8), and then perform the integrals, as it was done in [25]. This procedure becomes rapidly quite involved, since different pieces of the kernel contribute for different orders in $\epsilon$.

In the following, we give yet a different representation for the dressing kernel, which may be more useful for the strong coupling expansion. The equation (5.4) can be written as

$$
\hat{K}_{d}\left(t, t^{\prime}\right)=-\frac{4}{\pi} \sum_{n \geq 0} \int_{-\infty}^{\infty} d t^{\prime \prime} \hat{K}_{-}\left(t, t^{\prime \prime}\right) e^{-2 n \epsilon\left|t^{\prime \prime}\right|} \hat{K}_{+}\left(-t^{\prime \prime}, t^{\prime}\right)
$$


This formula can be easily Fourier transformed back such that we get

$$
K_{d}\left(u, u^{\prime}\right)=-8 \sum_{n \geq 1} \int_{-\infty}^{\infty} d v K_{-}^{1, n}(u, v) K_{+}^{n, 1}\left(v, u^{\prime}\right)
$$

with

$$
\begin{aligned}
K_{\mp}^{m, n}(u, v) & =-\frac{1}{2 \pi i} \partial_{u} \sum_{\substack{l>0 ; \text { even } \\
\text { odd }}} \frac{1}{l}\left(\left(x^{(+m)}(u) x^{(-n)}(v)\right)^{-l}-\left(x^{(-m)}(u) x^{(+n)}(v)\right)^{-l}\right), \\
x^{( \pm n)}(u) & =u \pm i n \epsilon+\sqrt{(u \pm i n \epsilon)^{2}-1} .
\end{aligned}
$$

The variables $x^{( \pm n)}$ appeared in [13], where they play an important role in defining the dressing phase. They live on a torus with modulus defined by

$$
\frac{k_{n}^{\prime}}{k_{n}}=n \epsilon
$$

and they are associated with the bound states of magnons [35].

The expression (5.11) for the dressing kernel involves a sum of integrals along an infinite set of contours $\mathcal{C}_{n \epsilon}^{\infty}, n=1,2,3, \ldots$, depicted in Fig. 5, defined in the same way as the contour $\mathcal{C}^{\infty}$, but with $\epsilon$ replaced by $n \epsilon$

$$
\mathcal{C}_{n \epsilon}^{\infty}=\{x \in \mathbb{C} \mid(x-\bar{x})(1-1 / x \bar{x})=4 i n \epsilon, \quad \bar{x} x>1\} .
$$
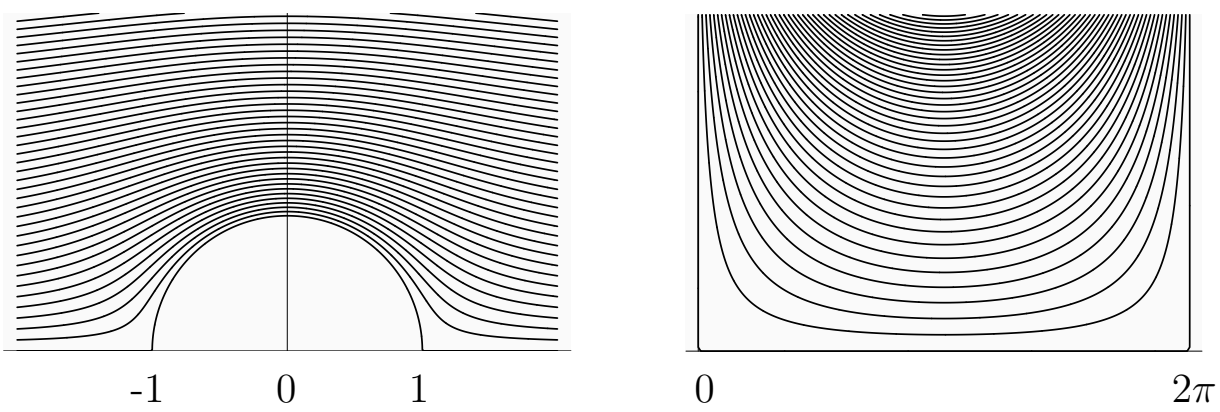

Fig. 5: The sequence of contours in the definition of the dressing kernel drown in the $x$-plane (left) and in the $(p+i \beta)$-plane (right).

In the strong coupling limit, the expression (5.11) becomes tractable, since we can evaluate the sum using the Euler-Maclaurin formula

$$
\sum_{n=1}^{\infty} f(n \epsilon)=\frac{1}{\epsilon} \int_{0}^{\infty} f(z) d z-\frac{1}{2} f(0)-\sum_{k=1}^{\infty} \epsilon^{2 k-1} \frac{B_{2 k}}{(2 k) !} f^{(2 k-1)}(0) .
$$


We thus extend $n \epsilon$ to the continuous variable

$$
n \epsilon=z
$$

and define the contour $\mathcal{C}_{z}^{\infty}$ by

$$
\mathcal{C}_{z}^{\infty}: \quad \sin \frac{p}{2} \sinh \frac{\beta}{2}=z, \quad \beta>0 .
$$

The integrals over $v$ and the variable $z$ become a double integral over the domain $\mathcal{D}$ in the complex $x$-plane spanned by the contours $\mathcal{C}_{z}^{\infty}$. The domain in question is the the upper half plane minus the unit semi-disk. In terms of the complexified momentum $\beta+i p=2 \ln x$, the domain $\mathcal{D}$ is the semi-infinite cylinder $\mathcal{D}=\{0 \leq p<2 \pi, \beta>0\}$.

The integral can be done without specifying a particular parametrization of the contour $\mathcal{C}_{z}^{\infty}$ if we express the integrand as a wedge product of differential forms. We first write the basic kernel as a 1-form

$$
K\left(x, x_{1}\right)=-\frac{d p}{4 \pi}+\frac{1}{4 \pi} \frac{\sinh \frac{\beta+\beta_{1}}{2} d p-\sin \frac{p-p_{1}}{2} d \beta}{\cosh \frac{\beta+\beta_{1}}{2}-\cos \frac{p-p_{1}}{2}} .
$$

Then the "even" kernel $K_{+}$is a one-form in the basis of $d p$ and $d \beta$,

$$
K_{+}\left(x, x_{2}\right)=\frac{1}{2 \pi} \frac{\sinh \frac{\beta+\beta_{2}}{2} \cos \frac{p-p_{2}}{2} d p-\sin \frac{p-p_{2}}{2} \cosh \frac{\beta+\beta_{2}}{2} d \beta}{\cosh \left(\beta+\beta_{2}\right)-\cos \left(p-p_{2}\right)},
$$

while the "odd" kernel $K_{-}$is a zero-form in this basis,

$$
K_{-}\left(x_{1}, x\right)=-\frac{1}{4 \pi} d p_{1}+\frac{1}{4 \pi} \frac{d p_{1} \sinh \left(\beta+\beta_{1}\right)-d \beta_{1} \sin \left(p_{1}-p\right)}{\cosh \left(\beta+\beta_{1}\right)-\cos \left(p_{1}-p\right)} .
$$

Also, for the differential $d z$ we have from (5.16)

$$
d z=\frac{1}{2} \sinh (\beta / 2) \cos (p / 2) d p+\frac{1}{2} \sin (p / 2) \cosh (\beta / 2) d \beta .
$$

Then the leading term in the strong coupling limit $\epsilon \rightarrow 0$ is given by

$$
K_{d}^{0}\left(x_{1}, x_{2}\right)=-\frac{8}{\epsilon} \int_{0}^{\infty} d z \int_{x \in \mathcal{C}_{z}} K_{-} K_{+} \equiv-\frac{8}{\epsilon} \int_{\mathcal{D}} K_{-} d z \wedge K_{+} .
$$

The evaluation of this integral (the calculation is sketched in Appendix B) leads to the following expression for $K_{d}^{0}$

$$
\begin{aligned}
K_{d}^{0}\left(x_{1}, x_{2}\right) & =\frac{1}{4 \pi \epsilon} \partial_{u}\left[-\tilde{\chi}\left(x_{1}^{-}, x_{2}^{+}\right)+\tilde{\chi}\left(x_{2}^{+}, x_{1}^{-}\right)+\tilde{\chi}\left(x_{1}^{-},-x_{2}^{+}\right)-\tilde{\chi}\left(-x_{2}^{+}, x_{1}^{-}\right)+\text {c.c. }\right], \\
\tilde{\chi}(x, y) & =-\left(x+\frac{1}{x}\right) \log \left(y-\frac{1}{x}\right) .
\end{aligned}
$$

This expression must be antisymmetrized with respect to $x_{2}^{ \pm} \rightarrow x_{2}^{\mp}$ (or, equivalently, $\left.p_{2} \rightarrow-p_{2}, \beta_{2} \rightarrow \beta_{2}\right)$. After antisymmetrization the result coincides 14 with the AFS kernel $K^{A F S}(u, v)$, symmetrized with respect to $v \rightarrow-v$.

\footnotetext{
${ }^{14}$ It is useful to notice that we can substitute $\chi_{0}$ with $\tilde{\chi}$ to calculate AFS term in (5.23).
} 


\subsection{The strong coupling limit from the AFS kernel}

To compute the leading order(s) of the dressing kernel, we do not really need the integral representation derived in the previous subsection. It is more straightforward to use the strong coupling representation of the dressing phase $\theta_{12}=\theta\left(u_{1}, u_{2}\right)$ worked out in [13, 14]. The latter can be cast in the form

$$
\begin{aligned}
\theta_{12} & =\frac{1}{2 \epsilon}\left[\chi_{12}^{--}-\chi_{12}^{+-}-\chi_{12}^{-+}+\chi_{12}^{++}-(1 \leftrightarrow 2)\right] \\
\chi_{12}^{r s} & =\chi\left(x_{1}^{r}, x_{2}^{s}\right), \quad r, s= \pm
\end{aligned}
$$

where the function $\chi$ can be expanded in powers of the inverse coupling constant

$$
\chi=\sum_{n \geq 0} \chi_{n}(2 \epsilon)^{n}
$$

To compute the leading term in the anomalous dimension, the AFS term [11] is sufficient,

$$
\chi_{0}(x, y)=-\frac{x y-1}{y} \ln \frac{x y-1}{x y},
$$

while for the next correction we will need the Hernández-López [12, 54] term

$$
\begin{aligned}
& \chi_{1}(x, y)=\frac{1}{\pi}\left[\ln \frac{y-1}{y+1} \ln \frac{x-1 / y}{x-y}\right. \\
& \left.+\operatorname{Li}_{2} \frac{\sqrt{y}-1 / \sqrt{y}}{\sqrt{y}-\sqrt{x}}-\operatorname{Li}_{2} \frac{\sqrt{y}+1 / \sqrt{y}}{\sqrt{y}-\sqrt{x}}+\operatorname{Li}_{2} \frac{\sqrt{y}-1 / \sqrt{y}}{\sqrt{y}+\sqrt{x}}-\operatorname{Li}_{2} \frac{\sqrt{y}+1 / \sqrt{y}}{\sqrt{y}+\sqrt{x}}\right] .
\end{aligned}
$$

In the following, we give the first non-zero order of the dressing AFS phase. The kernel can be simply obtained by taking the derivative of the phase divided by $2 \pi$, with the right sign. We find, for the sectors " $<$ " and " $>$ ",

$$
\begin{aligned}
& \theta^{>>}\left(u_{1}, u_{2}\right)=\frac{2 \epsilon}{\sinh \beta_{1} / 2 \sinh \beta_{2} / 2} \frac{\sinh \left(\beta_{1}-\beta_{2}\right) / 4}{\sinh \left(\beta_{1}+\beta_{2}\right) / 4}-2 \varphi^{>>}\left(u_{1}, u_{2}\right)+\mathcal{O}\left(\epsilon^{2}\right), \\
& \theta^{><}\left(u_{1}, u_{2}\right)=-\frac{2 \sin p_{2} / 2}{\sinh \beta_{1} / 2}-2 \varphi^{><}\left(u_{1}, u_{2}\right)+\mathcal{O}(\epsilon) \\
& \theta^{<>}\left(u_{1}, u_{2}\right)=\frac{2 \sin p_{1} / 2}{\sinh \beta_{2} / 2}-2 \varphi^{<>}\left(u_{1}, u_{2}\right)+\mathcal{O}(\epsilon), \\
& \theta^{<<}\left(u_{1}, u_{2}\right)=\frac{1}{\epsilon}\left[\left(\cos p_{1} / 2-\cos p_{2} / 2\right) \ln \frac{\sin ^{2}\left(p_{1}-p_{2}\right) / 4}{\sin ^{2}\left(p_{1}+p_{2}\right) / 4}\right]+\mathcal{O}(1) .
\end{aligned}
$$

We remind thet $\varphi$ is the $s u(1 \mid 1)$ phase.

In the sectors " + " and " - ", where $\beta$ and $p$ (or $2 \pi-p$ ) are of order $\sqrt{\epsilon}$, we introduce the parameter $\alpha$, which is of order one, as follows

$$
\begin{aligned}
& p_{i}=2 \sqrt{\epsilon} \alpha_{i}, \quad \beta_{i}=2 \sqrt{\epsilon} \alpha_{i}^{-1} \text { with } \alpha_{i}>0 \quad \text { in the region " }+", \\
& p_{i}=2 \pi+2 \sqrt{\epsilon} \alpha_{i}, \quad \beta_{i}=-2 \sqrt{\epsilon} \alpha_{i}^{-1} \quad \text { with } \alpha_{i}<0 \quad \text { in the region " }-" .
\end{aligned}
$$


Then we have

$$
\begin{aligned}
\theta^{ \pm \pm}\left(\alpha_{1}, \alpha_{2}\right)= & \pm \frac{1}{2}\left[\left(\alpha_{1}^{-2}-\alpha_{1}^{2}-\alpha_{2}^{-2}+\alpha_{2}^{2}\right) \ln \frac{\left(\alpha_{1}^{-1}+\alpha_{2}^{-1}\right)^{2}+\left(\alpha_{1}-\alpha_{2}\right)^{2}}{\left(\alpha_{1}^{-1}+\alpha_{2}^{-1}\right)^{2}+\left(\alpha_{1}+\alpha_{2}\right)^{2}},\right. \\
& \left.-4 i \ln \frac{\alpha_{1}^{-1}+\alpha_{2}^{-1}-i\left(\alpha_{1}-\alpha_{2}\right)}{\alpha_{1}^{-1}+\alpha_{2}^{-1}+i\left(\alpha_{1}-\alpha_{2}\right)}\right]+\mathcal{O}(\sqrt{\epsilon}), \\
\theta^{ \pm \mp}\left(\alpha_{1}, \alpha_{2}\right)= & \pm 2 \alpha_{1} \alpha_{2}+\mathcal{O}(\sqrt{\epsilon}) \\
\theta^{ \pm>}\left(\alpha_{1}, u_{2}\right)= & \pm 2 \sqrt{\epsilon} \frac{\alpha_{1}}{\sinh \beta_{2} / 2}+\mathcal{O}(\epsilon), \\
\theta^{ \pm<}\left(\alpha_{1}, u_{2}\right)= & -\frac{2}{\sqrt{\epsilon}} \alpha_{1} \sin p_{2} / 2+\mathcal{O}(1), \\
\theta^{> \pm}\left(u_{1}, \alpha_{2}\right)= & \mp 2 \sqrt{\epsilon} \frac{\alpha_{2}}{\sinh \beta_{1} / 2}+\mathcal{O}(\epsilon), \\
\theta^{< \pm}\left(u_{1}, \alpha_{2}\right)= & \frac{2}{\sqrt{\epsilon}} \alpha_{2} \sin p_{1} / 2+\mathcal{O}(1) .
\end{aligned}
$$

In (5.27) and (5.28) we omitted the signs coming from $u$ being in $u>1$ and $u<-1$. A mnemonic rule to reproduce these signs is to take $\operatorname{sign} \beta_{i}=\operatorname{sign} u_{i}$. In the hyperbolic parametrization with the variable $s$, which we give below, the signs are automatically taken into account

$$
\begin{aligned}
& \theta^{>>}\left(s_{1}, s_{2}\right)=2 \epsilon \sinh s \sinh s^{\prime} \tanh \frac{s-s^{\prime}}{2}-2 \varphi^{>>}\left(s_{1}, s_{2}\right)+\mathcal{O}\left(\epsilon^{2}\right) \\
& \theta^{><}\left(s_{1}, s_{2}\right)=-\frac{2 \sinh s_{1}}{\cosh s_{2}}-2 \varphi^{><}\left(s_{1}, s_{2}\right)+\mathcal{O}(\epsilon) \\
& \theta^{<>}\left(s_{1}, s_{2}\right)=\frac{2 \sinh s_{2}}{\cosh s_{1}}-2 \varphi^{<>}\left(s_{1}, s_{2}\right)+\mathcal{O}(\epsilon) \\
& \theta^{<<}\left(s_{1}, s_{2}\right)=\frac{2}{\epsilon}\left(\frac{\sinh \left(s_{1}-s_{2}\right)}{\cosh s_{1} \cosh s_{2}} \ln \left|\tanh \frac{s_{1}-s_{2}}{2}\right|\right)+\mathcal{O}(1) .
\end{aligned}
$$

In the regimes where one of the variables is in the region " + " or " - ", we put $\alpha_{i}= \pm e^{ \pm s_{i}}$

$$
\begin{aligned}
\theta^{ \pm \pm}\left(s_{1}, s_{2}\right)= & \mp\left[\left(\sinh 2 s_{1}-\sinh 2 s_{2}\right) \ln \frac{1+e^{ \pm 2\left(s_{1}+s_{2}\right)} \tanh ^{2} \frac{s_{1}-s_{2}}{2}}{1+e^{ \pm 2\left(s_{1}+s_{2}\right)}}\right. \\
& \left.+4 \arctan \left(e^{ \pm\left(s_{1}+s_{2}\right)} \tanh \frac{s_{1}-s_{2}}{2}\right)\right]+\mathcal{O}(\sqrt{\epsilon}), \\
\theta^{ \pm \mp}\left(s_{1}, s_{2}\right)= & \mp 2 e^{ \pm\left(s_{1}-s_{2}\right)}+\mathcal{O}(\sqrt{\epsilon}),
\end{aligned}
$$




$$
\begin{aligned}
& \theta^{ \pm>}\left(s_{1}, s_{2}\right)=2 \sqrt{\epsilon} e^{ \pm s_{1}} \sinh s_{2}+\mathcal{O}(\epsilon) \\
& \theta^{ \pm<}\left(s_{1}, s_{2}\right)=\mp \frac{2}{\sqrt{\epsilon}} \frac{e^{ \pm s_{1}}}{\cosh s_{2}}+\mathcal{O}(1) \\
& \theta^{> \pm}\left(s_{1}, s_{2}\right)=-2 \sqrt{\epsilon} e^{ \pm s_{2}} \sinh s_{1}+\mathcal{O}(\epsilon), \\
& \theta^{< \pm}\left(s_{1}, s_{2}\right)= \pm \frac{2}{\sqrt{\epsilon}} \frac{e^{ \pm s_{2}}}{\cosh s_{1}}+\mathcal{O}(1) .
\end{aligned}
$$

It is important to note that, in what concerns $\theta^{ \pm \pm}$, the equation (5.30) is not the correct answer, since in this region all terms $\chi_{n}$ in (5.24) contribute to the $\mathcal{O}(1)$ order. This was pointed out by Maldacena and Swanson [28], who derived an integral representation for the scattering phase in this region.

It is interesting to note that $\theta^{ \pm \pm}$and $\theta^{ \pm \mp}$ in eq. (5.30) depend naturally on the rotated coordinates

$$
s=s_{1}+s_{2}, \quad \tilde{s}=s_{1}-s_{2},
$$

and that for large values of $s$, positive or negative, we have

$$
\theta^{++}\left(s_{1}, s_{2}\right) \simeq 2 e^{s}\left(\tanh \frac{\tilde{s}}{2}-1-\sinh \tilde{s}\right) .
$$

\section{Solving the integral equations in the strong cou- pling limit with the dressing kernel}

Once we have obtained the leading term of the dressed kernels, we are able to compute the leading contribution to the energy of the states we are interested in. In the $s u(1 \mid 1)$ and $s u(2)$ sectors, we do not need to obtain the functional form for the density explicitly. This is a fortunate situation, since in the $( \pm \pm)$ regime, where most of the roots are concentrated, we do not know the kernel in a closed form. The computation is very similar to the one performed in [11], where the $\lambda^{1 / 4}$ behavior for the anomalous dimensions was first derived.

\subsection{The $s u(1 \mid 1)$ dressed case}

The results of the numerical and analytical computation by Beccaria et al. [39, 40] for the energy of the highest excited state in the $s u(1 \mid 1)$ sector can be also derived from the integral equation. As it was shown in [40], the same results can be obtained using the light-cone Bethe ansatz equations [55], first derived in the $s u(1 \mid 1)$ sector by Arutynov and Frolov [56]. In this sector, the total kernel is

$$
\mathcal{K}=K+K_{d} .
$$

First, we can show that the densities $\rho_{<}$and $\rho_{>}$are subdominant, while the main contribution comes from $\rho_{ \pm}$. The density in the "giant magnons" region is given by the equation

$$
\rho^{<}=\frac{1}{2 \pi} \frac{d p}{d s}+\mathcal{K}^{<<} \rho^{<}+\mathcal{K}^{<>} \rho^{>}+\sum_{ \pm} \mathcal{K}^{< \pm} \rho^{ \pm}
$$


By symmetry, we have $\rho_{+}(\alpha)=\rho_{-}(-\alpha)$ and since

$$
\int_{0}^{\infty} \alpha \rho_{+}(\alpha) d \alpha+\int_{-\infty}^{0} \alpha \rho_{-}(\alpha) d \alpha=0
$$

we conclude that the \pm terms in (6.2) cancel

$$
\rho^{<}=\frac{1}{2 \pi} \frac{d p}{d s}+\mathcal{K}^{<<} \rho^{<}+\mathcal{K}^{<>} \rho^{>}+\mathcal{O}(1) .
$$

All the terms except $\mathcal{K}^{<<} \rho^{<}$are of the order 1 . Since $\mathcal{K}^{<<}=\mathcal{O}(1 / \epsilon)$ we deduce that $\rho_{<}(s)=\mathcal{O}(\epsilon)$. Similarly, we can write

$$
\rho^{>}=\frac{1}{2 \pi} \frac{d p}{d s}+\mathcal{K}^{><} \rho^{<}+\mathcal{K}^{>>} \rho^{>}+\sum_{ \pm} \mathcal{K}^{> \pm} \rho^{ \pm} .
$$

Again the leading term in the sum vanishes and all the terms in the r.h.s. are of the order $\epsilon$, so that $\rho_{>}(s)$ is also of order at most $\epsilon$. Therefore the roots of the Bethe equations must concentrate in the regions \pm , or "near-flat space" region. In this region, we have

$$
\rho^{ \pm}=\frac{1}{2 \pi} \frac{d p}{d \alpha}+\mathcal{K}^{ \pm<} \rho^{<}+\mathcal{K}^{ \pm>} \rho^{>}\left(s^{\prime}\right)+\mathcal{K}^{ \pm \pm} \rho^{ \pm}+\mathcal{K}^{ \pm \mp} \rho^{\mp}
$$

At the leading order, we are left with

$$
\rho^{ \pm}(\alpha)=\int d \alpha^{\prime} \mathcal{K}^{ \pm \pm}\left(\alpha, \alpha^{\prime}\right) \rho^{ \pm}\left(\alpha^{\prime}\right)+\int d \alpha^{\prime} \mathcal{K}^{ \pm \mp}\left(\alpha, \alpha^{\prime}\right) \rho^{\mp}\left(\alpha^{\prime}\right)
$$

or, by specializing to the sign + and by integrating over $\alpha$,

$$
k^{+}(\alpha)=-\frac{1}{2 \pi} \int_{0}^{\infty} d \alpha^{\prime} \theta_{\text {tot }}^{++}\left(\alpha, \alpha^{\prime}\right) \rho^{+}\left(\alpha^{\prime}\right)-\frac{1}{2 \pi} \int_{-\infty}^{0} d \alpha^{\prime} \theta_{\text {tot }}^{+-}\left(\alpha, \alpha^{\prime}\right) \rho^{-}\left(\alpha^{\prime}\right),
$$

where $k^{+}(\alpha)$ is the counting function, $k^{+}(\alpha) \equiv \int_{0}^{\alpha} d \alpha \rho^{+}(\alpha)$. $\theta_{\text {tot }}^{+ \pm}$is defined as $\theta_{\text {tot }}^{+ \pm}=$ $-2 \pi \int_{0}^{\alpha} d \alpha \mathcal{K}^{+ \pm}$and at leading order is equal to the dressing part $\theta^{+ \pm}$. By multiplication with $\rho(\alpha)$ and integration we get

$$
\begin{aligned}
\int_{0}^{\infty} d \alpha k^{+}(\alpha) \rho^{+}(\alpha) & =-\frac{1}{2 \pi} \int_{0}^{\infty} d \alpha \int_{0}^{\infty} d \alpha^{\prime} \theta^{++}\left(\alpha, \alpha^{\prime}\right) \rho^{+}(\alpha) \rho^{+}\left(\alpha^{\prime}\right) \\
& -\frac{1}{2 \pi} \int_{0}^{\infty} d \alpha \int_{-\infty}^{0} d \alpha^{\prime} \theta^{+-}\left(\alpha, \alpha^{\prime}\right) \rho^{+}(\alpha) \rho^{-}\left(\alpha^{\prime}\right)
\end{aligned}
$$

Here we used that $\theta^{+-}\left(0, \alpha^{\prime}\right)=\theta^{++}\left(0, \alpha^{\prime}\right)=0$. Let us remind that $\alpha=0$ corresponds to $u=\infty$. Due to the anti-symmetry of the phase $\theta^{++}$, the first integral in the r.h.s. of (6.8) vanishes, while the l.h.s. is equal to

$$
\int_{0}^{\infty} d \alpha k^{+}(\alpha) \rho^{+}(\alpha)=\int_{0}^{1 / 2} d k^{+} k^{+}=\frac{1}{8} .
$$


Finally, we use that $\theta^{+-}\left(\alpha, \alpha^{\prime}\right)=2 \alpha \alpha^{\prime}$ and the symmetry $\rho^{+}(\alpha)=\rho^{-}(-\alpha)$ to cast the equation (6.8) in the form

$$
\int_{0}^{\infty} d \alpha \alpha \rho^{+}(\alpha)=\sqrt{\frac{\pi}{8}} .
$$

This is all we need to compute the leading order in the energy

$$
E_{s u(1 \mid 1)}^{d}=4 g L \int e^{-\beta / 2} \sin p / 2 \rho(p) d p \simeq 4 \sqrt{g} L \int_{0}^{\infty} d \alpha \alpha \rho^{+}(\alpha)=\sqrt{2 \pi g} L .
$$

It is remarkable that the energy of this state is of the order $\lambda^{1 / 4}$. It would be interesting to know if there are states in the $s u(1 \mid 1)$ sector which have greater energy. This would be possible for states where the fraction of giant magnons is larger than $1 / \sqrt{g}$. Presumably, the strong repulsion among the giant magnons will prevent this phenomenon to happen.

\subsection{The $\mathrm{su}(2)$ dressed case}

The computation of the leading order in the energy for $s u(2)$ sector goes similarly to that for $s u(1 \mid 1)$, with two differences. First, there is a non-trivial contribution from the $s u(2)$ kernel to the leading order of $\theta_{\text {tot }}^{++}$

$$
\theta_{s u(2)}\left(u, u^{\prime}\right)=2 \pi \int_{\infty}^{u} d v K_{s u(2)}\left(v, u^{\prime}\right)=2 \int_{u}^{\infty} d v \frac{2 \epsilon}{\left(v-u^{\prime}\right)^{2}+4 \epsilon^{2}}=\pi+\theta_{\text {asym }}\left(u, u^{\prime}\right) .
$$

Therefore, the first term in the r.h.s. of the equation (6.8) does not cancel anymore and we obtain

$$
\int_{0}^{\infty} d \alpha k^{+}(\alpha) \rho^{+}(\alpha)=-\frac{1}{2}\left(\int_{0}^{\infty} d \alpha \rho^{+}(\alpha)\right)^{2}+\frac{1}{\pi}\left(\int_{0}^{\infty} d \alpha \alpha \rho^{+}(\alpha)\right)^{2} .
$$

Second, the maximum of counting function is $1 / 4$ instead of $1 / 2$, because the total number of magnons is $M=L / 2$, and

$$
\int_{0}^{\infty} d \alpha \rho^{+}(\alpha)=\int_{0}^{1 / 4} d k^{+}=\frac{1}{4}
$$

From equation (6.13) we obtain

$$
\int_{0}^{\infty} d \alpha \alpha \rho^{+}(\alpha)=\sqrt{\frac{\pi}{16}}
$$

which reproduces 15 the value of the energy obtained by Beccaria et al. [39, 40,

$$
E_{s u(2)}^{d}=\sqrt{\pi g} L+\mathcal{O}\left(g^{0}\right) .
$$

\footnotetext{
${ }^{15}$ We thank M. Beccaria for an email exchange which helped us to remedy a discrepancy with their results in the first version of this paper.
} 


\subsection{The $\operatorname{sl}(2)$ dressed case}

For the $s l(2)$ case, the total kernel with the $s u(2)$ piece subtracted, as defined in (2.11), is equal to

$$
\mathcal{K}\left(u, u^{\prime}\right)=2 K\left(u, u^{\prime}\right)+K_{d}\left(u, u^{\prime}\right) \equiv \frac{1}{2 \pi} \partial_{u} \phi\left(u, u^{\prime}\right)
$$

The BES equation for this sector takes the simplest form when written for the shifted density function

$$
\bar{\sigma}(u) \equiv \sigma(u)-\frac{2 \epsilon}{\pi}
$$

which, up to a negative factor, equals the total density at the leading order in $S$, see (2.16). The BES equation reads

$$
\bar{\sigma}(u)=\frac{1}{\pi} \int_{-\infty}^{\infty} d u^{\prime} \frac{2 \epsilon \bar{\sigma}\left(u^{\prime}\right)}{\left(u-u^{\prime}\right)^{2}+4 \epsilon^{2}}+\int d u^{\prime} \mathcal{K}\left(u, u^{\prime}\right) \bar{\sigma}\left(u^{\prime}\right) .
$$

We are interested in expanding equation (6.18) in powers of $\epsilon$. At small $\epsilon$

$$
\frac{2 \epsilon}{\pi} \int_{-\infty}^{\infty} d u^{\prime}\left(\frac{\bar{\sigma}\left(u^{\prime}\right)}{\left(u-u^{\prime}\right)^{2}}+\mathcal{O}\left(\epsilon^{2}\right)\right)+\int d u^{\prime} \mathcal{K}\left(u, u^{\prime}\right) \bar{\sigma}\left(u^{\prime}\right)=0
$$

This equation splits into several equations, which couple the different regions in $u$. For instance, if $|u|<1$ we have

$$
\begin{aligned}
& \frac{2 \epsilon}{\pi}\left(f_{-1}^{1} d u^{\prime} \frac{\bar{\sigma}^{<}\left(u^{\prime}\right)}{\left(u-u^{\prime}\right)^{2}}+\int_{>} d u^{\prime} \frac{\bar{\sigma}^{>}\left(u^{\prime}\right)}{\left(u-u^{\prime}\right)^{2}}+\mathcal{O}\left(\epsilon^{2}\right)\right)+\int_{-1}^{1} d u^{\prime} \mathcal{K}^{<<}\left(u, u^{\prime}\right) \bar{\sigma}^{<}\left(u^{\prime}\right) \\
& +\int_{>} d u^{\prime} \mathcal{K}^{<>}\left(u, u^{\prime}\right) \bar{\sigma}^{>}\left(u^{\prime}\right)+\sum_{ \pm} \int d \alpha \mathcal{K}^{< \pm}(u, \alpha) \bar{\sigma}^{ \pm}(\alpha)=0
\end{aligned}
$$

Equation (6.18) was analyzed in the first two orders in $\epsilon$ by the authors of [25]. They did not consider the regions around $u= \pm 1$, which can contain a fraction of the roots. Equation (6.18) suggests that the $\epsilon$-expansion of the density starts at order $\epsilon$. The structure of the leading terms (5.28) in the expansion of the dressing kernel, where half-integer powers of $\epsilon$ appear, suggests that the density and the anomalous dimension may involve a half-integer powers of $\epsilon$ as well. However, these corrections seem to appear in higher orders in the anomalous dimension, and this may explain why they have not been seen yet 16 In the following, we are going to ignore the corrections coming from the vicinity of the points $u= \pm 1$ and solve perturbatively the equation (6.18), by considering the decomposition of the density and the kernel in integer powers of $\epsilon$

$$
\begin{aligned}
\bar{\sigma}(u) & =\sigma(u)-\frac{2 \epsilon}{\pi}=\epsilon \bar{\sigma}_{1}(u)+\epsilon^{2} \sigma_{2}(u)+\epsilon^{3} \sigma_{3}(u)+\ldots, \\
\mathcal{K}\left(u, u^{\prime}\right) & =\epsilon^{-1} \mathcal{K}_{-1}\left(u, u^{\prime}\right)+\mathcal{K}_{0}\left(u, u^{\prime}\right)+\epsilon \mathcal{K}_{1}\left(u, u^{\prime}\right)+\ldots
\end{aligned}
$$

\footnotetext{
${ }^{16}$ As far we can see, the first non-zero order for $\sigma^{ \pm}(\alpha)$ is at most $\epsilon^{2}$, and there may be corrections of order $\epsilon^{5 / 2}$ to $\sigma^{<}(u)$ and of order $\epsilon^{3 / 2}$ for $\bar{\sigma}^{>}(u)$. The last of these corrections would induce a term of order $g^{1 / 2}$ in the anomalous dimension. To decide whether they are here or not we have to go to higher orders in the expansion of the kernel.
} 
- order $\epsilon^{0}$

At the leading order in $\epsilon$ equation (6.20) reads, in simplified notations,

$$
\mathcal{K}_{-1} \bar{\sigma}_{1}=0
$$

To this order only the $<<$ sector contributes

$$
\int_{-1}^{1} d u^{\prime} \mathcal{K}_{-1}^{<<}\left(u, u^{\prime}\right) \bar{\sigma}_{1}^{<}\left(u^{\prime}\right)=0
$$

It was proven in [25] that the kernel $\mathcal{K}_{-1}^{<<}$is non-degenerate, therefore we have $\bar{\sigma}_{1}^{<}(u)=0$, or equivalently $\sigma_{1}=2 / \pi$.

- order $\epsilon^{1}$

The $O(\epsilon)$ term in the expansion of equation (6.20) relates $\bar{\sigma}_{1}$ and $\sigma_{2}$

$$
\mathcal{K}_{-1}^{<<} \sigma_{2}^{<}+\mathcal{K}_{0}^{<>} \bar{\sigma}_{1}^{>}=0
$$

The kernel in the second term is antisymmetric in $u^{\prime} \rightarrow-u^{\prime}$

$$
\mathcal{K}_{0}^{<>}\left(u, u^{\prime}\right)=-\frac{1}{\pi} \partial_{u} \frac{\sqrt{1-u^{2}}}{\sqrt{u^{\prime 2}-1}} \operatorname{sgn} u^{\prime}
$$

so that it vanishes upon integration against the symmetric function $\bar{\sigma}_{1}^{>}(u)$. As pointed out in [25], the subleading density $\sigma_{2}^{<}(u)$ vanishes as well. It is worth noticing that $\mathcal{K}_{0}^{<>}\left(u, u^{\prime}\right)$ is the sum of two terms, one coming from the $s u(1 \mid 1)$ kernel and the other from the dressing phase, $\mathcal{K}_{0}^{<>}\left(u, u^{\prime}\right)=2 K_{0}^{<>}\left(u, u^{\prime}\right)+K_{d, 0}^{<>}\left(u, u^{\prime}\right)$.

\section{- order $\epsilon^{2}$}

At this order we have an equation relating $\bar{\sigma}_{1}, \sigma_{2}$ and $\sigma_{3}$

$$
\frac{2}{\pi} f_{-\infty}^{\infty} d u^{\prime} \frac{\bar{\sigma}_{1}\left(u^{\prime}\right)}{\left(u-u^{\prime}\right)^{2}}+\mathcal{K}_{-1} \sigma_{3}+\mathcal{K}_{0} \sigma_{2}+\mathcal{K}_{1} \bar{\sigma}_{1}=0 .
$$

○ The interval $|u|>1$

Consider first the region $|u|>1$, where the equation takes the form

$$
\frac{2}{\pi} f_{>} d u^{\prime} \frac{\bar{\sigma}_{1}^{>}\left(u^{\prime}\right)}{\left(u-u^{\prime}\right)^{2}}+\int_{>} d u^{\prime} \mathcal{K}_{1}^{>>}\left(u, u^{\prime}\right) \bar{\sigma}_{1}^{>}\left(u^{\prime}\right)=0
$$

with the kernel $\mathcal{K}_{1}^{>>}\left(u, u^{\prime}\right) \equiv \frac{1}{2 \pi} \partial_{u} \phi_{1}$ given by (5.29). Equation (6.25) is a total derivative, so we first integrate it to

$$
f_{>} d u^{\prime} \bar{\sigma}_{1}^{>}\left(u^{\prime}\right)\left(-\frac{2}{\pi} \frac{1}{u-u^{\prime}}+\frac{1}{2 \pi} \phi_{1}\left(u, u^{\prime}\right)\right)=0 .
$$


As the phase is anti-symmetric, there is no integration constant.

At this point, we find it more useful to switch to the variable $s$, defined by $u=\operatorname{coth} s$, and (6.26) becomes

$$
\frac{1}{\pi} f_{-\infty}^{\infty} d s^{\prime} \bar{\sigma}_{1}^{>}\left(s^{\prime}\right) \sinh s^{\prime} \operatorname{coth} \frac{s-s^{\prime}}{2}=0
$$

Now we can reformulate the integral equation (6.26) as a Riemann boundary value problem. We first rewrite (6.27) in terms of the normalizable density $\sigma_{1}=\bar{\sigma}_{1}+2 / \pi$

$$
\frac{1}{\pi} f_{-\infty}^{\infty} d s^{\prime} \sigma_{1}^{>}\left(s^{\prime}\right) \sinh s^{\prime} \operatorname{coth} \frac{s-s^{\prime}}{2}=\frac{4 s}{\pi^{2} \sinh s} .
$$

Then, after the redefinition

$$
\omega=e^{s}, \quad r(\omega)=-r(1 / \omega)=\sigma_{1}^{>}(s) \sinh s,
$$

the l.h.s. of (6.28) takes the form of a Cauchy integral

$$
\int_{0}^{\infty} \frac{d \omega^{\prime}}{\omega^{\prime}} r\left(\omega^{\prime}\right)+2 f_{0}^{\infty} d \omega^{\prime} \frac{r\left(\omega^{\prime}\right)}{\omega-\omega^{\prime}}=\frac{8}{\pi} \frac{\ln \omega}{\omega-\omega^{-1}}, \quad(\omega>0) .
$$

The first term on the 1.h.s. is actually zero due to the anti-symmetry of $r(\omega)$.

Equation (6.30) can be formulated in terms of a boundary condition for the resolvent

$$
R(\omega)=R(1 / \omega)=\int_{0}^{\infty} d \omega^{\prime} \frac{r\left(\omega^{\prime}\right)}{\omega-\omega^{\prime}}
$$

which has a cut on the positive axis, namely,

$$
R(\omega+i 0)+R(\omega-i 0)=\frac{8}{\pi} \frac{\ln \omega}{\omega-\omega^{-1}} \quad(\omega>0) .
$$

The most general solution of (6.32) with the symmetry $R(\omega)=R(1 / \omega)$ is of the form

$$
R(\omega)=\frac{4}{\pi} \frac{\ln (-\omega)+\sqrt{-\omega} Q(\omega)-\sqrt{-1 / \omega} Q(1 / \omega)}{\omega-1 / \omega},
$$

where $Q(\omega)$ is a rational function. The latter is determined by the analytical properties of the resolvent (no poles outside the positive real axis) and the requirement that the density $\sigma_{1}$ is normalizable

$$
\int_{>} d u \sigma_{1}(u)=\int_{-\infty}^{\infty} d s \sigma_{1}^{>}(s)=\int_{0}^{\infty} d \omega \frac{2 r(\omega)}{\omega^{2}-1}=\text { finite. }
$$

The only solution with these properties corresponds to $Q=-1 / 2$ :

$$
R(\omega)=\frac{4}{\pi} \frac{\ln (-\omega)-\frac{1}{2}(\sqrt{-\omega}-\sqrt{-1 / \omega})}{\omega-1 / \omega},
$$


and its discontinuity along the positive real axis,

$$
r(\omega)=\frac{R(\omega+i 0)-R(\omega-i 0)}{2 \pi i}=\frac{2}{\pi}\left(\frac{2}{\omega-1 / \omega}-\frac{\sqrt{\omega}}{\omega-1}\right),
$$

reproduces the solution found by Alday et al [25]:

$$
\sigma_{<}(u)=\epsilon \sigma_{1}^{<}=\frac{1}{2 \pi g}, \quad \sigma_{>}(u)=\epsilon \sigma_{1}^{>}(u)=\frac{1}{2 \pi g}\left(1-\cosh \frac{s}{2}\right) .
$$

The total integral of the density,

$$
\int_{-\infty}^{\infty} d s\left(\sigma^{<}(s)+\sigma^{>}(s)\right)=\frac{1}{\pi g}+\frac{\pi-4}{4 \pi g}=\frac{1}{4 g},
$$

reproduces the correct leading behavior for the twist-two anomalous dimension

$$
f(g)=4 g+\ldots .
$$

○ The interval $|u|<1$

Let us assume for the moment that the corrections from the vicinity of the points $u= \pm 1$ can be neglected. Then, in the region $|u|<1$, the equation will reduce to

$$
\frac{2}{\pi} \int_{\left|u^{\prime}\right|>1} d u^{\prime} \frac{\bar{\sigma}_{1}^{>}\left(u^{\prime}\right)}{\left(u-u^{\prime}\right)^{2}}+\mathcal{K}_{-1}^{<<} \sigma_{3}^{<}+\mathcal{K}_{1}^{<>} \bar{\sigma}_{1}^{>}=0 .
$$

The kernels in the first and the third term combine to

$$
\mathcal{K}_{1}^{<>}\left(u, u^{\prime}\right)+\frac{2}{\pi} \frac{1}{\left(u-u^{\prime}\right)^{2}}=\frac{1}{\pi} \partial_{u} \frac{\sinh s^{\prime} \cosh s+\frac{2}{\pi}\left(s-s^{\prime}\right) \sinh ^{2} s^{\prime}}{\cosh \left(s-s^{\prime}\right)} .
$$

The procedure to obtain the previous expression was the following: we expressed the first two terms $\chi_{0}$ and $\chi_{1}$ in the dressing phase in terms of the variables $p$ and $\beta^{\prime}$ and expanded them up to the desired order in $\epsilon$. Then we expressed the results in terms of the variables $s, s^{\prime}$ by using the relations

$$
u=\cos p / 2=\tanh s, \quad u^{\prime}=\cosh \beta^{\prime} / 2=\operatorname{coth} s^{\prime} .
$$

Integrating the product of the kernel (6.40) with $\bar{\sigma}_{1}^{>}=-2 \cosh (s / 2)$ gives zero, so we deduce that

$$
\sigma_{3}^{<}(u)=0
$$

- order $\epsilon^{3}$

At this order, equation (6.20) gives

$$
\frac{2}{\pi} f_{-\infty}^{\infty} d u^{\prime} \frac{\sigma_{2}\left(u^{\prime}\right)}{\left(u-u^{\prime}\right)^{2}}+\mathcal{K}_{-1} \sigma_{4}+\mathcal{K}_{0} \sigma_{3}+\mathcal{K}_{1} \sigma_{2}+\mathcal{K}_{2} \bar{\sigma}_{1}=0
$$


The subleading density $\sigma_{2}^{>}(u)$, which we need in order to compute the subleading term in $f(g)$, is supposed to be determined by the equation with $|u|>1$

$$
\frac{2}{\pi} f_{>} d u^{\prime} \frac{\sigma_{2}^{>}\left(u^{\prime}\right)}{\left(u-u^{\prime}\right)^{2}}+\mathcal{K}_{1}^{>>} \sigma_{2}^{>}=-\mathcal{K}_{2}^{>>} \bar{\sigma}_{1}^{>}
$$

After integrating with respect to $u$ and passing to the $s$ parametrization, this equation takes the same form as (6.28), but with different inhomogeneous term

$$
\frac{1}{\pi} f_{-\infty}^{\infty} d s^{\prime} \sigma_{2}^{>}\left(s^{\prime}\right) \sinh s^{\prime} \operatorname{coth} \frac{s-s^{\prime}}{2}=-\frac{1}{2 \pi \sinh s} \int_{-\infty}^{\infty} d s^{\prime} \bar{\sigma}_{1}^{>}\left(s^{\prime}\right) \phi_{2}^{>>}\left(s, s^{\prime}\right) .
$$

The inhomogeneous term in the r.h.s. can be computed from the expression

$$
\phi_{2}^{>>}\left(s, s^{\prime}\right)=\frac{4\left(\sinh s \sinh s^{\prime}\right)^{2}}{\pi} \frac{\left(s-s^{\prime}\right) \cosh \left(s-s^{\prime}\right)-\sinh \left(s-s^{\prime}\right)}{\sinh ^{2}\left(s-s^{\prime}\right)},
$$

which, after being integrated with $\bar{\sigma}_{1}^{>}(s)=-\frac{2}{\pi} \cosh (s / 2)\left|u^{\prime}(s)\right|$ gives

$$
-\frac{1}{2 \pi \sinh s} \int_{-\infty}^{\infty} d s^{\prime} \bar{\sigma}_{1}^{>}\left(s^{\prime}\right) \phi_{2}^{>>}\left(s, s^{\prime}\right)=-\frac{2}{\pi} \sinh s \sinh (s / 2) .
$$

After the redefinition (6.29) we again reduce the integral equation to a Riemann boundary value problem

$$
R(\omega+i 0)+R(\omega-i 0)=2 f_{0}^{\infty} d \omega^{\prime} \frac{r\left(\omega^{\prime}\right)}{\omega-\omega^{\prime}}=-\frac{\sqrt{\omega}(\omega-1)\left(\omega^{2}-1\right)}{2 \omega^{2}} .
$$

The solution is

$$
R(s)=-\frac{s \sinh s \cosh (s / 2)}{2 \pi}, \quad r(s)=\frac{s \sinh s \sinh (s / 2)}{2 \pi^{2}},
$$

which gives for the $\epsilon^{2}$-correction to the density

$$
\sigma_{2}^{>}(s)=\frac{s \sinh (s / 2)}{2 \pi^{2}} .
$$

This correction to the density grows exponentially at $s \rightarrow \infty$ and is not normalizable 17 . The significance of the result (6.47) is that the higher order corrections to the density become large in the vicinity of the points $u= \pm 1$, which correspond to $s= \pm \infty$. Therefore, in order to determine the next correction to the scaling function $f(g)$ we have to take into account the contribution coming from the points $u= \pm 1$ (the regimes \pm ). The equations starting with (6.39) should be corrected accordingly. We leave this problem for future work.

\footnotetext{
${ }^{17}$ Strictly speaking, the maximum value of $s$ to which we should integrate is $s_{\max }=\mathrm{K} / 2 \simeq-\ln \epsilon$. If we stop the integral at $s_{\max }$, we will obtain a contribution to the universal scaling function of the order $\ln \epsilon / \sqrt{\epsilon}$, which violates the hypothesis that the expansion is in integer power of $\epsilon$. Also, it signals that the limit $\epsilon \rightarrow 0$ is not uniform, and this is in accordance with the fact that in the near-flat space regime all the terms $\chi_{n}$ contribute [28].
} 


\section{Outlook}

We presented a method to treat the strong coupling limit of the Bethe ansatz equations, which can be used to extract the spectrum of anomalous dimensions. We found it useful to express the spectral parameter appearing in the Bethe ansatz equations by means of a complexified momentum, with real part $p$ and imaginary part $\beta$

$$
x^{ \pm}=e^{\beta / 2 \pm i p / 2} .
$$

It is worth noticing that these variables already appeared in [37], where the BDS magnons were interpreted as bound states of more fundamental, fermionic excitations. There, $p$ is the momentum of the bound states, while $\beta$ is related to the "size" of the bound state.

Furthermore, the functions of $p$ and $\beta$ are naturally expressed in terms of elliptic functions. Our elliptic parametrization is a version of that in [10]. In the strong coupling limit, the elliptic parametrization degenerates into a hyperbolic one. In order to take into account properly the different regimes, we used different expansions of the elliptic functions in terms of hyperbolic functions, depending on the value of the the elliptic parameter $s$ : around $s=0, \mathrm{~K}$ and $\pm \mathrm{K} / 2$. These regimes correspond to the "planewave" [32], the "giant magnons" [33, 34] and the "near-flat space" regions, recently characterized in [28], respectively. It is interesting to note that the momentum $p$ and the energy $\varepsilon \equiv E / 4 g$ of the excitations have, in the plane wave and near-flat space regime, relativistic-like expressions

$$
\begin{array}{lcl}
p=\epsilon \sinh s & \varepsilon=\epsilon(\cosh s-1) & \text { (plane wave) } \\
p= \pm 2 \sqrt{\epsilon} e^{ \pm s} & \varepsilon=2 \sqrt{\epsilon} e^{ \pm s} & \text { (near flat space) }
\end{array}
$$

with $s$ playing the role of the rapidity variable. However, only the positive branch of the energy, corresponding to particles, appear in the sectors we consider, sectors which should be closed at any order in perturbation theory. It is important to understand how the antiparticle branch will appear; most probably by another copy of the sector which joins in. An example of how this should happen is offered by the $s u(2)$ principal chiral model [57, 58], where the particles and antiparticles correspond to the two copies of $s u(2)$-symmetric excitations.

The strong coupling limit of the kernels without the dressing factor is relatively simple. The giant magnons interact through a delta term, typical for the statistical repulsion. In the $s u(1 \mid 1)$ sector, there is also a "length-changing" term. The excitations of the plane-wave type do not interact with each other, at leading order, but they interact with the giant magnons. The excitations in the near-flat space regime do not contribute to the leading term.

The strong coupling limit of the dressing kernel is more involved. For the giant magnons and plane-wave regions, we can do it safely with the strong coupling expansion of the dressing kernel [11, 12, 13, at least for the first few orders in $\epsilon$. For the near-flat space limit, however, as it was pointed out in [28], all the terms in the strong coupling series contribute to the leading order.

When we consider the kernels with the dressing phase, the repulsion of the giant magnons is so large that their density vanishes in the leading order. For $s u(1 \mid 1)$ and $s u(2)$ 
sectors, most of the magnons are concentrated in the near-flat space regime. Fortunately, although we do not have an explicit expression of the kernel in this regime, it is possible to obtain the energy for the highest excited state at leading order.

The most exciting application of our method is to obtain the strong coupling expansion of the twist-two operator anomalous dimension and to compare with the string computations [21, 22]. Several recent analytical and numerical works [23, 24, 25] were devoted to this task. In particular, 25] obtained the leading term, using the Fourier transform representation. They also tried to obtain the result by a method very similar to ours, and found that the first two orders of the equation are not sufficient to fix the leading order. Here, we show that it is possible to obtained the leading order, by going an order higher. We also show that, in order to compute the higher correction, we have to take into account the contribution from the near-flat space regime.

Note added. An integral representation of the dressing phase, seemingly related to (1.5), was used in [59] to explore the analytic properties of the $S$-matrix .

\section{Acknowledgments}

D.S. and I.K. thank the Albert Einstein Institute, Potsdam, and Kavli Institute for Theoretical Physics, Santa Barbara, where part of this work was done, for hospitality. D.S. thanks Matthias Staudacher for collaboration in early stages of this project. We thank Gleb Arutyunov, Matteo Beccaria, Marcus Benna, Nick Dorey, Burkhard Eden, Sergey Frolov, Igor Klebanov, Lev Lipatov, Matthias Staudacher and Arkady Tseytlin for helpful discussions and Stefan Zieme for spotting many typos in the first version of the paper. This work has been partially supported by the National Science Foundation under Grant No. PHY99-07949, by the European Union through ENRAGE network (contract MRTNCT-2004-005616) and the by ANR programs GIMP (contract ANR-05-BLAN-0029-01) and INT-AdS/CFT (contract ANR36ADSCSTZ)

\section{A The elliptic parametrization}

The modulus of the elliptic map is

$$
k=\frac{1}{\sqrt{1+\epsilon^{2}}}, \quad k^{\prime}=\frac{\epsilon}{\sqrt{1+\epsilon^{2}}} .
$$

The real and imaginary parts of the complexified momentum

$$
p(s)=2 k^{\prime} \int_{0}^{s} \frac{d v}{\operatorname{dn} v}, \quad \beta(s)=\ln \frac{1+k^{\prime}}{1-k^{\prime}}-2 \int_{K}^{s} \operatorname{cs} v d v
$$

have the symmetries

$$
\begin{aligned}
p(2 K-u) & =2 \pi-p(u), & & \beta(2 K-u)=\beta(u) \\
p(u) & =-p(-u), & & \beta(-u)=\beta(u) \mp 2 i \pi .
\end{aligned}
$$

The phase in the Bethe equations depends on $\beta$ and $p$ through

$$
\cosh \frac{1}{2} \beta=\frac{1}{k} \mathrm{~ns} s, \quad \sinh \frac{1}{2} \beta=\frac{1}{k} \mathrm{ds} s,
$$




$$
\begin{gathered}
\cos \frac{1}{2} p=\operatorname{cd} s, \quad \sin \frac{1}{2} p=k^{\prime} \operatorname{sd} s . \\
e^{ \pm \beta / 2}=\frac{1 \pm \operatorname{dn} s}{k \operatorname{sn} s}, \quad e^{ \pm i p / 2}=\operatorname{cd} s \pm i k^{\prime} \operatorname{sd} s \\
\partial_{s} p=2 k^{\prime} \operatorname{nd} s, \quad \partial_{s} \beta=-2 \operatorname{cs} s .
\end{gathered}
$$

Below we give asymptotic expressions of these functions in the limit $k^{\prime} \simeq \epsilon \rightarrow 0$, where

$$
K \approx \ln \frac{4}{\epsilon}, \quad K^{\prime} \approx \frac{\pi}{2}, \quad \tilde{q}=e^{-\pi K / K^{\prime}} \approx \frac{\epsilon^{2}}{16} .
$$

In this limit it is appropriate to use the expansions of the three elliptic functions in terms of hyperbolic functions of $v=\pi s / 2 K^{\prime}$ :

$$
\begin{aligned}
\operatorname{sn} s & =\frac{\pi}{2 k K^{\prime}}\left(\tanh v+4 \sum_{n=1}^{\infty}(-)^{n} \frac{\tilde{q}^{2 n}}{1+\tilde{q}^{2 n}} \sinh 2 n v\right) \\
\operatorname{cn} s & =\frac{\pi}{2 k K^{\prime}}\left(\frac{1}{\cosh v}+4 \sum_{n=1}^{\infty}(-)^{n} \frac{\tilde{q}^{2 n-1}}{1+\tilde{q}^{2 n-1}} \cosh (2 n-1) v\right) \\
\operatorname{dn} s & =\frac{\pi}{2 K^{\prime}}\left(\frac{1}{\cosh v}-4 \sum_{n=1}^{\infty}(-)^{n} \frac{\tilde{q}^{2 n-1}}{1-\tilde{q}^{2 n-1}} \cosh (2 n-1) v\right) .
\end{aligned}
$$

In the limit $\epsilon \rightarrow 0$ and up to $o\left(\epsilon^{4}\right)$ terms the three functions are given by [45]

$$
\begin{aligned}
\operatorname{sn} s & \simeq \tanh s+\epsilon^{2} \frac{(\sinh s \cosh s-s)}{4 \cosh ^{2} s} \\
\operatorname{cn} s & \simeq \frac{1}{\cosh s}-\epsilon^{2} \frac{\tanh s}{4}\left(\sinh s-\frac{s}{\cosh s}\right) \\
\operatorname{dn} s & \simeq \frac{1}{\cosh s}+\epsilon^{2} \frac{\tanh s}{4}\left(\sinh s+\frac{s}{\cosh s}\right) .
\end{aligned}
$$

We used only the leading term; it gives a good approximation for $s \in I^{>}=\left[-\frac{1}{2} K, \frac{1}{2} K\right]$. Similarly, for the interval $s \in I^{<}$we can use the approximation

$$
\begin{aligned}
\operatorname{sn}(\mathrm{K}-s) & \simeq \operatorname{cd} s \\
\operatorname{cn}(\mathrm{K}-s) \simeq k^{\prime} \mathrm{sd} s & \simeq \epsilon \sinh s \\
\operatorname{dn}(\mathrm{K}-s) & \simeq k^{\prime} \operatorname{nd} s \simeq \epsilon \cosh s
\end{aligned}
$$

For the functions that enter in the definition of the kernel we find:

(a) In the interval $I^{>}$:

$$
\begin{array}{r}
\cosh (\beta / 2)=\operatorname{coth} s, \quad \sinh (\beta / 2)=\frac{1}{\sinh s}, \quad \beta^{\prime}=-\frac{2}{\sinh s}, \\
\cos (p / 2)=1, \quad \sin (p / 2)=k^{\prime} \sinh s, \quad \partial_{s} p=2 k^{\prime} \cosh s
\end{array}
$$




$$
\begin{array}{r}
u^{\prime}=-\frac{1}{\sinh ^{2} s}, \quad u=\operatorname{coth} s \\
e^{\beta / 2}=\operatorname{coth} \frac{s}{2}, \quad e^{s}=\sqrt{\frac{u+1}{u-1}}
\end{array}
$$

(b) In the interval $I^{<}$, after replacing $s \rightarrow K-s$ :

$$
\begin{array}{r}
\cosh (\beta / 2)=1, \quad \sin (\beta / 2)=k^{\prime} \cosh s, \quad \partial_{s} \beta=-2 k^{\prime} \sinh s, \\
\cos (p / 2)=\tanh s, \quad \sin (p / 2)=1 / \cosh s, \quad \partial_{s} p=2 / \cosh s \\
u^{\prime}=\frac{1}{\cosh ^{2} s}, \quad u=\tanh s, \\
e^{i p / 2}=\frac{1+i e^{-s}}{1-i e^{-s}}, \quad e^{s}=\sqrt{\frac{1-u}{1+u}}
\end{array}
$$

Plugging these expressions in (3.27) we evaluate the kernel in the four possible regimes. In the sector $s, s_{1} \in I^{>}$the numerator in (3.27) is of order $\epsilon$, while the denominator remains finite. Therefore

$$
K^{>>}\left(s, s_{1}\right)=0 .
$$

For the non-diagonal elements of (3.29) we obtain

$$
\begin{aligned}
K^{><}\left(s, s_{1}\right)=K\left(s, \mathrm{~K}-s_{1}\right) & \simeq \frac{1}{4 \pi} \frac{\beta^{\prime} \sin \left(p_{1} / 2\right)}{\cosh (\beta / 2)-\cos \left(p_{1} / 2\right)} \\
& \simeq \frac{1}{2 \pi} \frac{1}{\cosh \left(s-s_{1}\right)}, \\
K^{<>}\left(s, s_{1}\right)=K\left(\mathrm{~K}-s, s_{1}\right) & \simeq \frac{1}{4 \pi} p^{\prime}-\frac{1}{4 \pi} \frac{p^{\prime} \sinh \left(\beta_{1} / 2\right)}{\cosh \left(\beta_{1} / 2\right)-\cos (p / 2)} \\
& \simeq \frac{1}{2 \pi} \frac{1}{\cosh s}-\frac{1}{2 \pi} \frac{1}{\cosh \left(s-s_{1}\right)} .
\end{aligned}
$$

Finally, if both arguments are in $I^{<}$, then the kernel $K^{<<}\left(s, s_{1}\right)=K\left(\mathrm{~K}-s, \mathrm{~K}-s_{1}\right)$ vanishes except near the double pole of the denominator at $s=s_{1}$, where it can be approximated by a delta-function:

$$
\begin{aligned}
K^{<<}\left(s, s_{1}\right)=K\left(\mathrm{~K}-s, \mathrm{~K}-s_{1}\right) & \simeq \frac{1}{4 \pi} p^{\prime}-\frac{1}{2 \pi} \frac{p^{\prime} \beta}{\beta^{2}+\sin ^{2}\left(p-p_{1}\right) / 2} \\
& \simeq \frac{1}{4 \pi} p^{\prime}-\left|\partial_{s} p\right| \delta\left(p-p_{1}\right)
\end{aligned}
$$




$$
\simeq \frac{1}{2 \pi} \frac{1}{\cosh s}-\delta\left(s-s_{1}\right)
$$

- The regime $s \simeq \pm K / 2$

Assume that $s= \pm K / 2+y$, where $y \ll \sqrt{\epsilon}$. We first evaluate the three basic Jacobi elliptic functions for the shifted argument:

$$
\begin{aligned}
& \frac{\operatorname{sn}\left(s \pm \frac{1}{2} \mathrm{~K}\right)}{\mathrm{sn} \frac{1}{2} \mathrm{~K}}=\frac{k^{\prime} \mathrm{sd} \pm \mathrm{cn}}{\mathrm{cn}^{2}+k^{\prime} \mathrm{sn}^{2}}(y) \\
& \frac{\mathrm{cn}\left(s \pm \frac{1}{2} \mathrm{~K}\right)}{\mathrm{cn} \frac{1}{2} \mathrm{~K}}=\frac{\mathrm{cn} \mp \operatorname{sn} \mathrm{dn}}{\mathrm{cn}^{2}+k^{\prime} \mathrm{sn}^{2}}(y) \\
& \frac{\operatorname{dn}\left(s \pm \frac{1}{2} \mathrm{~K}\right)}{\operatorname{dn} \frac{1}{2} \mathrm{~K}}=\frac{\mathrm{dn} \mp\left(1-k^{\prime}\right) \operatorname{sn} \mathrm{cn}}{\mathrm{cn}^{2}+k^{\prime} \mathrm{sn}^{2}}(y) .
\end{aligned}
$$

When $k^{\prime} \approx \epsilon \rightarrow 0$, the values of the three functions at $s=\frac{1}{2} \mathrm{~K}$ are

$$
\begin{aligned}
& \operatorname{sn}\left(\frac{1}{2} \mathrm{~K}\right)=1 / \sqrt{1+k^{\prime}} \\
& \operatorname{cn}\left(\frac{1}{2} \mathrm{~K}\right)=\sqrt{k^{\prime} /\left(1+k^{\prime}\right)} \approx \sqrt{\epsilon}, \\
& \operatorname{dn}\left(\frac{1}{2} \mathrm{~K}\right)=\sqrt{k^{\prime}} \quad \approx \sqrt{\epsilon},
\end{aligned}
$$

so that

$$
\begin{aligned}
\operatorname{sn}\left( \pm \frac{1}{2} \mathrm{~K}+y\right) & = \pm\left(1-\frac{1}{2} \epsilon e^{\mp 2 y}\right) \\
\operatorname{cn}\left( \pm \frac{1}{2} \mathrm{~K}+y\right) & =\sqrt{\epsilon} e^{\mp y}\left(1-\epsilon \sinh ^{2} y\right) \\
\operatorname{dn}\left( \pm \frac{1}{2} \mathrm{~K}+y\right) & =\sqrt{\epsilon} e^{\mp y}(1 \pm \epsilon \sinh y \cosh y)
\end{aligned}
$$

From here one finds for the asymptotics of the functions $p(s), \beta(s)$ and $u(s)$ for $s= \pm \mathrm{K} / 2+y$ :

$$
\begin{aligned}
\sinh (\beta / 2) & = \pm \sqrt{\epsilon} e^{\mp y}\left(1+\frac{1}{2} \epsilon \cosh 2 y\right) \\
\sin (p / 2) & = \pm \sqrt{\epsilon} e^{ \pm y}\left(1-\frac{1}{2} \epsilon \cosh 2 y\right) \\
u & = \pm 1-\epsilon \sinh 2 y+o\left(\epsilon^{2}\right) .
\end{aligned}
$$

\section{B Evaluation of $K_{d}^{0}$}

Here we will evaluate the integral (5.21) for the strong coupling limit of $K_{d}$. We have

$$
\begin{aligned}
& K_{-}\left(x_{1}, x\right)=\frac{K_{-}^{p} d p_{1}+K_{-}^{\beta} d \beta_{1}}{4 \pi} \\
& K_{+}\left(x, x_{2}\right)=\frac{K_{+}^{p} d p+K_{+}^{\beta} d \beta}{2 \pi}
\end{aligned}
$$




$$
d z \quad=\frac{1}{2} Z^{p} d p+\frac{1}{2} Z^{\beta} d \beta
$$

where

$$
\begin{aligned}
& K_{-}^{p}=\frac{\cos \left(p-p_{1}\right)-e^{-\left|\beta+\beta_{1}\right|}}{H_{1}}, \quad K_{-}^{\beta}=-\frac{\sin \left(p_{1}-p\right)}{H_{1}} ; \\
& K_{+}^{p}=\frac{\sinh \frac{\beta+\beta_{2}}{2} \cos \frac{p-p_{2}}{2}}{H_{2}}, \quad K_{+}^{\beta}=-\frac{\sin \frac{p-p_{2}}{2} \cosh \frac{\beta+\beta_{2}}{2}}{H_{2}} ; \\
& Z^{p}=\sinh (\beta / 2) \cos (p / 2), \quad Z^{\beta}=\sin (p / 2) \cosh (\beta / 2) ; \\
& H_{a}=\cosh \left(\beta+\beta_{a}\right)-\cos \left(p-p_{a}\right), \quad a=1,2 .
\end{aligned}
$$

The integral to evaluate is

$$
\begin{aligned}
K_{d}^{0}\left(x_{1}, x_{2}\right) & =-\frac{1}{2 \pi^{2} \epsilon} \int_{0}^{2 \pi} d p \int_{0}^{\infty} d \beta\left(K_{-}^{p} d p_{1}+K_{-}^{\beta} d \beta_{1}\right)\left(Z^{p} K_{+}^{\beta}-Z^{\beta} K_{+}^{p}\right) \\
& =\frac{1}{4 \pi^{2} \epsilon} \int_{0}^{2 \pi} d p \int_{0}^{\infty} d \beta\left[A(p, \beta) d p_{1}+B(p, \beta) d \beta_{1}\right]
\end{aligned}
$$

where

$$
\begin{aligned}
A(\beta, p) & =\left(\cos \left(p-p_{1}\right)-e^{-\left|\beta+\beta_{1}\right|}\right) G(\beta, p) \\
B(\beta, p) & =-\sin \left(p_{1}-p\right) G(\beta, p) \\
G(\beta, p) & =\frac{\sin \left(p-\frac{p_{2}}{2}\right) \sinh \left(\beta+\frac{\beta_{2}}{2}\right)+\sinh \frac{\beta_{2}}{2} \sin \frac{p_{2}}{2}}{\left(\cosh \left(\beta+\beta_{1}\right)-\cos \left(p_{1}-p\right)\right)\left(\cosh \left(\beta+\beta_{2}\right)-\cos \left(p-p_{2}\right)\right)} .
\end{aligned}
$$

The strategy of calculation is the following: first we take an integral over $p$, which is of the form:

$$
I=\int_{0}^{2 \pi} d p R(\cos p, \sin p)
$$

where $R$ is a rational function. By symmetrization $p \rightarrow-p$ we reduce the integral to

$$
I=\int_{0}^{2 \pi} d p \tilde{R}\left(\cos p, \sin ^{2} p\right)
$$

and, after substituting $\cos p=-t$, it can be evaluated by taking the residues.

The integral over $\beta$ becomes, after the substitution $\beta=-\log x$, an integral from 0 to 1 of a rational expression and can also be performed explicitly. As a final result, we obtain

$$
\int_{0}^{\infty} d \beta \int_{0}^{2 \pi} d p A(p, \beta)=i \pi\left(\frac{1}{x_{2}^{-}}+\frac{1}{2}\left(x_{1}^{+}-\frac{1}{x_{1}^{+}}\right) \log \frac{1-\frac{1}{x_{1}^{+} x_{2}^{-}}}{1+\frac{1}{x_{1}^{+} x_{2}^{-}}}-\text {c.c. }\right)
$$




$$
\int_{0}^{\infty} d \beta \int_{0}^{2 \pi} d p B(p, \beta)=\pi\left(\frac{1}{x_{2}^{-}}+\frac{1}{2}\left(x_{1}^{+}-\frac{1}{x_{1}^{+}}\right) \log \frac{1-\frac{1}{x_{1}^{+} x_{2}^{-}}}{1+\frac{1}{x_{1}^{+} x_{2}^{-}}}+\text {c.c. }\right) .
$$

From here we deduce the expression for $K_{d}^{0}$, presented in (5.22).

\section{References}

[1] J. A. Minahan and K. Zarembo, "The Bethe-ansatz for $\mathcal{N}=4$ super Yang-Mills", JHEP 0303, 013 (2003), hep-th/0212208.

[2] N. Beisert and M. Staudacher, "The $\mathcal{N}=4 S Y M$ Integrable Super Spin Chain", Nucl. Phys. B670, 439 (2003),hep-th/0307042.

[3] N. Beisert, C. Kristjansen and M. Staudacher, "The Dilatation Operator of $\mathcal{N}=4$ Conformal Super Yang-Mills Theory", Nucl. Phys. B664, 131 (2003), hep-th/0303060.

[4] J. M. Maldacena, "The large $N$ limit of superconformal field theories and supergravity", Adv. Theor. Math. Phys. 2, 231 (1998), hep-th/9711200.

[5] S. S. Gubser, I. R. Klebanov and A. M. Polyakov, "Gauge theory correlators from non-critical string theory", Phys. Lett. B428, 105 (1998), hep-th/9802109.

[6] E. Witten, "Anti-de Sitter space and holography", Adv. Theor. Math. Phys. 2, 253 (1998), hep-th/9802150.

[7] M. Staudacher, "The factorized S-matrix of CFT/AdS," JHEP 0505 (2005) 054 hep-th/0412188.

[8] N. Beisert and M. Staudacher, "Long-range PSU(2,2-4) Bethe ansaetze for gauge theory and strings", Nucl. Phys. B 727, 1 (2005), hep-th/0504190.

[9] N. Beisert, "The Analytic Bethe Ansatz for a Chain with Centrally Extended su(2-2) Symmetry", J. Stat. Mech. 0701, P017 (2007), nlin.si/0610017.

[10] R. A. Janik, "The AdS(5) $\times S^{5}$ superstring worldsheet $S$-matrix and crossing symmetry", Phys. Rev. D 73, 086006 (2006), hep-th/0603038.

[11] G. Arutyunov, S. Frolov and M. Staudacher, "Bethe ansatz for quantum strings", JHEP 0410, 016 (2004), hep-th/0406256.

[12] R. Hernández and E. López, "Quantum corrections to the string Bethe ansatz", JHEP 0607, 004 (2006), hep-th/0603204.

[13] N. Beisert, R. Hernández and E. López, "A Crossing-Symmetric Phase for $A d S_{5} \times S^{5}$ Strings", hep-th/0609044.

[14] N. Beisert, B. Eden and M. Staudacher, "Transcendentality and crossing", J. Stat. Mech. 0701, P021 (2007), hep-th/0610251.

[15] A. V. Kotikov, L. N. Lipatov, A. I. Onishchenko and V. N. Velizhanin, "Three-loop universal anomalous dimension of the Wilson operators in $\mathcal{N}=4$ SUSY Yang-Mills model", Phys. Lett. B595, 521 (2004), hep-th/0404092.

[16] S. Moch, J. A. M. Vermaseren and A. Vogt, "The three-loop splitting functions in QCD: The non-singlet case", Nucl. Phys. B688, 101 (2004), hep-ph/0403192. 
[17] C. Anastasiou, Z. Bern, L. J. Dixon and D. A. Kosower, "Planar amplitudes in maximally supersymmetric Yang-Mills theory", Phys. Rev. Lett. 91, 251602 (2003), hep-th/0309040.

[18] Z. Bern, L. J. Dixon and V. A. Smirnov, "Iteration of planar amplitudes in maximally supersymmetric Yang-Mills theory at three loops and beyond", Phys. Rev. D72, 085001 (2005), hep-th/0505205.

[19] Z. Bern, M. Czakon, L. J. Dixon, D. A. Kosower and V. A. Smirnov, "The Four-Loop Planar Amplitude and Cusp Anomalous Dimension in Maximally Supersymmetric Yang-Mills Theory", hep-th/0610248.

[20] F. Cachazo, M. Spradlin and A. Volovich, "Four-loop cusp anomalous dimension from obstructions," hep-th/0612309.

[21] S. S. Gubser, I. R. Klebanov and A. M. Polyakov, "A semi-classical limit of the gauge/string correspondence", Nucl. Phys. B 636, 99 (2002), hep-th/0204051.

[22] S. Frolov and A. A. Tseytlin, "Semiclassical quantization of rotating superstring in $A d S_{5} \times S^{5} "$, JHEP 0206, 007 (2002), hep-th/0204226.

[23] A. V. Kotikov and L. N. Lipatov, "On the highest transcendentality in $N=4$ SUSY", hep-th/0611204.

[24] M. K. Benna, S. Benvenuti, I. R. Klebanov and A. Scardicchio, "A test of the AdS/CFT correspondence using high-spin operators", hep-th/0611135.

[25] L. F. Alday, G. Arutyunov, M. K. Benna, B. Eden and I. R. Klebanov, "On the strong coupling scaling dimension of high spin operators", hep-th/0702028.

[26] A. Rej, M. Staudacher and S. Zieme, "Nesting and dressing," hep-th/0702151.

[27] C. Gomez and R. Hernández, "Quantum deformed magnon kinematics," hep-th/0701200.

[28] J. Maldacena and I. Swanson, "Connecting giant magnons to the pp-wave: An interpolating limit of $A d S(5) \times S^{5}$ ", hep-th/0612079.

[29] B. Eden and M. Staudacher, "Integrability and transcendentality", J. Stat. Mech. 0611, P014 (2006), hep-th/0603157.

[30] N. Beisert, V. Dippel and M. Staudacher, "A Novel Long Range Spin Chain and Planar $\mathcal{N}=4$ Super Yang-Mills", JHEP 0407, 075 (2004), hep-th/0405001.

[31] G. P. Korchemsky, "Quasiclassical QCD pomeron", Nucl. Phys. B 462, 333 (1996), hep-th/9508025.

[32] D. Berenstein, J. M. Maldacena and H. Nastase, "Strings in flat space and pp waves from $\mathcal{N}=4$ Super Yang Mills", JHEP 0204, 013 (2002), hep-th/0202021.

[33] D. M. Hofman and J. M. Maldacena, "Giant magnons", J. Phys. A 39, 13095 (2006), hep-th/0604135.

[34] G. Arutyunov, S. Frolov and M. Zamaklar, "Finite-size effects from giant magnons," hep-th/0606126.

[35] N. Dorey, "Magnon bound states and the AdS/CFT correspondence", J. Phys. A 39 (2006) 13119 hep-th/0604175. 
[36] H. Y. Chen, N. Dorey and K. Okamura, "On the scattering of magnon boundstates," JHEP 0611, 035 (2006) : hep-th/0608047.

[37] A. Rej, D. Serban and M. Staudacher, "Planar $N=4$ gauge theory and the Hubbard model", JHEP 0603, 018 (2006), hep-th/0512077.

[38] K. Zarembo, "Antiferromagnetic operators in $N=4$ supersymmetric Yang-Mills theory", Phys. Lett. B 634, 552 (2006), hep-th/0512079.

[39] M. Beccaria and L. Del Debbio, "Bethe Ansatz solutions for highest states in $N=4$ $S Y M$ and AdS/CFT duality", JHEP 0609, 025 (2006), hep-th/0607236.

[40] M. Beccaria, G. F. De Angelis, L. Del Debbio and M. Picariello, "Highest states in light-cone $\operatorname{AdS}(5) \times S^{5}$ superstring", hep-th/0701167.

[41] D. Serban and M. Staudacher, unpublished, 2005

[42] L. D. Faddeev, "How Algebraic Bethe Ansatz works for integrable model", hep-th/9605187.

[43] G. Feverati, D. Fioravanti, P. Grinza and M. Rossi, "On the finite size corrections of anti-ferromagnetic anomalous dimensions in $N=4 S Y M$," JHEP 0605 (2006) 068 hep-th/0602189.

[44] G. Arutyunov and A. A. Tseytlin, "On highest-energy state in the su(1-1) sector of $N$ = 4 super Yang-Mills theory", JHEP 0605, 033 (2006), hep-th/0603113.

[45] P. Byrds and M. Friedman, Handbook of Elliptic Integrals for Engineers and Physicists, Springer-Verlag, Berlin 1954, p.25.

[46] E. H. Lieb and F. Y. Wu, "Absence of Mott transition in an exact solution of the short-range, one-band model in one dimension," Phys. Rev. Lett. 20 (1968) 1445; Erratum, ibid. 21 (1968) 192.

[47] F. D. M. Haldane, "Exact Jastrow-Gutzwiller Resonating Valence Bond Ground State Of The Spin 1/2 Antiferromagnetic Heisenberg Chain With $1 / R^{2}$ Exchange," Phys. Rev. Lett. 60, 635 (1988).

[48] B. S. Shastry, "Exact solution of an $S=1 / 2$ Heisenberg antiferromagnetic chain with long-ranged interactions", Phys. Rev. Lett. 60, 639 (1988).

[49] B. Sutherland, "Exact results for a quantum many body problem in one-dimension," Phys. Rev. A 4, 2019 (1971).

[50] S. N. M. Ruijsenaars and H. Schneider, "A New Class Of Integrable Systems And Its Relation To Solitons," Annals Phys. 170 (1986) 370.

[51] V. I. Inozemtsev, "Integrable Heisenberg-van Vleck chains with variable range exchange," Phys. Part. Nucl. 34 (2003) 166 [Fiz. Elem. Chast. Atom. Yadra 34 (2003) 332], hep-th/0201001.

[52] D. Serban and M. Staudacher, "Planar $\mathcal{N}=4$ gauge theory and the Inozemtsev long range spin chain", JHEP 0406, 001 (2004), hep-th/0401057.

[53] L. N. Lipatov, "Transcendentality and Eden-Staudacher equation", Talk at Workshop on Integrability in Gauge and String Theory, AEI, Potsdam, Germany, July 24-28, 2006, http://int06.aei.mpg.de/presentations/lipatov.pdf.

[54] G. Arutyunov and S. Frolov, "On $A d S_{5} \times S^{5}$ string S-matrix", Phys. Lett. B639, 378 (2006), hep-th/0604043. 
[55] G. Arutyunov, S. Frolov, J. Plefka and M. Zamaklar, "The Off-shell Symmetry Algebra of the Light-cone AdS $S_{5} \times S^{5}$ Superstring", hep-th/0609157.

[56] G. Arutyunov and S. Frolov, 'Uniform light-cone gauge for strings in AdS(5) $x S^{* *} 5$ : Solving su(1-1) sector," JHEP 0601 (2006) 055 hep-th/0510208.

[57] J. A. Minahan, "The SU(2) sector in AdS/CFT," Fortsch. Phys. 53 (2005) 828 hep-th/0503143.

[58] N. Gromov and V. Kazakov, "Asymptotic Bethe ansatz from string sigma model on $S^{3} \times R, "$ hep-th/0605026.

[59] N. Dorey, D. M. Hofman and J. Maldacena, "On the singularities of the magnon S-matrix," hep-th/0703104. 